\title{
Gas-free Calibrated fMRI with a Correction for Vessel-Size Sensitivity
}

\author{
Avery J.L. Berman ${ }^{1,2^{*}}$, Erin L. Mazerolle ${ }^{2,3}$, M. Ethan MacDonald ${ }^{2,3}$, \\ Nicholas P. Blockley ${ }^{4}$, Wen-Ming Luh ${ }^{5}$, G. Bruce Pike ${ }^{1-3,6}$ \\ ${ }^{1}$ Department of Biomedical Engineering, McGill University, Montreal, QC, Canada \\ ${ }^{2}$ Hotchkiss Brain Institute, University of Calgary, Calgary, AB, Canada \\ ${ }^{3}$ Department of Radiology, University of Calgary, Calgary, AB, Canada \\ ${ }^{4}$ FMRIB Centre, Nuffield Department of Clinical Neurosciences, \\ University of Oxford, Oxford, United Kingdom \\ ${ }^{5}$ Cornell MRI Facility, Cornell University, Ithaca, NY, USA \\ ${ }^{6}$ Department of Clinical Neuroscience, University of Calgary, Calgary, AB, Canada
}

\section{*Corresponding Author:}

Avery J.L. Berman

Health Sciences Centre, room 2910E 3330 Hospital Drive NW

Calgary, Alberta, Canada T2N 4N1

E-mail: avery.berman@mail.mcgill.ca 


\begin{abstract}
Calibrated functional magnetic resonance imaging ( $\mathrm{fMRI}$ ) is a method to independently measure the metabolic and hemodynamic contributions to the blood oxygenation level dependent (BOLD) signal. This technique typically requires the use of a respiratory challenge, such as hypercapnia or hyperoxia, to estimate the calibration constant, $M$. There has been a recent push to eliminate the gas challenge from the calibration procedure using asymmetric spin echo (ASE) based techniques. This study uses simulations to better understand spin echo (SE) and ASE signals, analytical modelling to characterize the signal evolution, and in vivo imaging to validate the modelling. Using simulations, it is shown how ASE imaging generally underestimates $M$ and how this depends on several parameters of the acquisition, including echo time and ASE offset, as well as the vessel size. This underestimation is the result of imperfect SE refocusing due to diffusion of water through the extravascular environment surrounding the microvasculature. By empirically characterizing this SE attenuation as an exponential decay that increases with echo time, we have proposed a quadratic ASE biophysical signal model. This model allows for the characterization and compensation of the SE attenuation if SE and ASE signals are acquired at multiple echo times. This was tested in healthy subjects and was found to significantly increase the estimates of $M$ across grey matter. These findings show promise for improved gas-free calibration and can be extended to other relaxation-based imaging studies of brain physiology.
\end{abstract}

\title{
Keywords
}

Calibrated fMRI; BOLD; Asymmetric spin echo; relaxometry; diffusion; cerebral metabolic rate of oxygen 


\section{Introduction}

Calibrated functional magnetic resonance imaging (fMRI) was developed to disentangle the hemodynamic and metabolic contributions to the blood oxygenation level dependent (BOLD) signal using simultaneous measurements of the gradient echo BOLD signal and cerebral blood flow (CBF) (Davis et al., 1998; Hoge et al., 1999). A calibration experiment is run to estimate the calibration constant, $M$, and is most commonly performed using a respiratory challenge where subjects inhale a gas mixture with additional carbon dioxide and/or oxygen to elicit changes in the BOLD signal and CBF or arterial oxygen tension (Bulte et al., 2012; Chiarelli et al., 2007; Davis et al., 1998; Gauthier and Hoge, 2012). The use of hypercapnia, the state of elevated $\mathrm{CO}_{2}$ in blood, suffers from multiple limitations: it may violate the assumption of isometabolism on which standard calibration models depend (Bulte et al., 2009; Chen and Pike, 2010a; Hall et al., 2011; Xu et al., 2011), and it typically measures perfusion changes using timeresolved arterial spin labelling (ASL), an imaging technique with a low signal-to-noise ratio. Similarly, the use of hyperoxia, the state of elevated $\mathrm{O}_{2}$ in blood, requires either the additional measurement of the oxygen extraction fraction and the concentration of hemoglobin in blood or the assumption of those two parameters (Chiarelli et al., 2007; Mark et al., 2011) that can lead to bias (Blockley et al., 2012). Hyperoxia may also produce concomitant decreases in CBF if blood $\mathrm{CO}_{2}$ is not controlled (Bulte et al., 2007; Croal et al., 2015). In general, gas challenges require additional apparatus and increased subject tolerance and preparation, thus, a gas-free alternative would greatly improve the appeal of calibrated fMRI.

To date, a limited number of studies have examined gas-free calibration of the BOLD signal by substituting the gas challenge with a measurement of $R_{2}{ }^{\prime}$ at rest, the reversible component of the transverse relaxation rate (Blockley et al., 2015; Fujita et al., 2006; Kida et al., 2000; Shu et al., 2015). Under the assumption that the primary difference between the apparent and the irreversible relaxation rates $\left(R_{2}{ }^{*}\right.$ and $R_{2}$, respectively) in a voxel is from the field inhomogeneities generated by deoxyhemoglobin (deoxyHb) (Blockley et al., 2012; Fujita et al., 2006), $R_{2}{ }^{\prime}$ is the favoured candidate for gas-free calibration due to its intimate relationship with baseline blood oxygen saturation and the deoxygenated cerebral blood volume (CBV) (Yablonskiy and Haacke, 1994). However, as in most areas of MR relaxometry, the apparent 
values of $R_{2}^{\prime}$ are highly dependent on the measurement technique and may produce different values due to multi-exponential decay, imperfect spin echo refocusing, and other acquisition related factors (Ni et al., 2014). Blockley et al. (2015) recently proposed a calibration technique that is insensitive to multi-exponential decay and intrinsic tissue $T_{2}$ differences based on using spin echo (SE) and asymmetric spin echo (ASE) imaging. When compared against traditional hypercapnic calibration, their ASE calibration underestimated $M$ across grey matter (GM) and the visual cortex, on average. This underestimation was postulated to arise from incomplete spin echo refocusing of spins diffusing in the extravascular space. This effect is the same source of contrast in SE BOLD imaging, and is known to be vessel-size and field strength dependent (Boxerman et al., 1995).

In addition to imperfect SE refocusing, several other sources may confound the observed $R_{2}{ }^{\prime}$ values. Macroscopic field inhomogeneities, which are prominent around air-tissue interfaces, lead to dramatic geometric distortions and signal intensity distortions in echo planar imaging (EPI). The intensity distortions tend to increase $R_{2}{ }^{\prime}$ and these effects can be mitigated by a range of acquisition-related methods (Blockley and Stone, 2016). Cerebrospinal fluid (CSF) has recently been shown to significantly increase measured $R_{2}$ ' in grey matter (Simon et al., 2016; Stone and Blockley, 2016). This is postulated to arise from a chemical shift between CSF and parenchyma, resulting in enhanced signal dephasing in tissue voxels with partial voluming with CSF (He and Yablonskiy, 2007). By adding a fluid attenuated inversion recovery (FLAIR) preparation to the imaging sequence, the CSF signal can be eliminated and the $R_{2}{ }^{\prime}$ of neighbouring parenchymal voxels tends to decrease. Unlike field inhomogeneities and CSF, which can be prospectively and retrospectively managed, additional non-deoxyHb sources of tissue magnetic susceptibility, such as iron depositions and myelin, will alter $R_{2}^{\prime}$ in a less predictable manner (they can increase or decrease $R_{2}^{\prime}$, depending on their susceptibility and relative concentration). Kida et al. (2000) found that these other sources of susceptibility have negligible contributions to the observed $R_{2}$ and $R_{2}{ }^{*}$ (and hence $R_{2}{ }^{\prime}$ ) at $7 \mathrm{~T}$ in rats. In this study, which was performed at a field strength of $3 \mathrm{~T}$, we do not consider these other sources, consistent with earlier work (Blockley et al., 2015; Fujita et al., 2003). 
The purpose of this study was to determine how incomplete refocusing of SE and ASE signals affects the estimation of $R_{2}{ }^{\prime}$ and how it can be accounted for to obtain a more accurate estimate of $M$. Simulations were used to determine the vessel-size dependence of the $R_{2}{ }^{\prime}$ underestimation and to develop a strategy to retrospectively correct for it. This strategy was tested in vivo, taking precautions to avoid confounds from macroscopic field inhomogeneities and CSF partial volume. These ASE-based $M$ calculations were compared against hypercapnic calibration in the same subjects.

\section{Theory}

\subsection{Calibrated fMRI with Asymmetric Spin Echo Imaging}

The standard calibrated fMRI model that relates changes in the cerebral metabolic rate of oxygen $\left(\mathrm{CMRO}_{2}\right)$ and $\mathrm{CBF}$ to changes in the gradient echo (GE) BOLD signal is (Davis et al., 1998)

$$
\frac{\Delta \mathrm{BOLD}}{\mathrm{BOLD}_{0}}=M\left(1-\left(\frac{\mathrm{CBF}}{\mathrm{CBF}_{0}}\right)^{\alpha-\beta}\left(\frac{\mathrm{CMRO}_{2}}{\mathrm{CMRO}_{2 \mid 0}}\right)^{\beta}\right)
$$

where the subscript ' 0 ' refers to a value at baseline and $\triangle \mathrm{BOLD}=\mathrm{BOLD}-\mathrm{BOLD}_{0} . \alpha$ is the Grubb constant and accounts for coupling between CBV and CBF arising from an empirical power law relation between the two (Grubb et al., 1974). $\beta$ describes the non-linear dependence of the change in $R_{2}{ }^{*}$ on the susceptibility offset of blood relative to tissue (Berman and Pike, 2016; Boxerman et al., 1995). $M$ is proportional to the resting concentration of deoxyHb in blood and it can be considered the maximum fractional increase in the GE BOLD signal, which would theoretically occur upon removal of all deoxyHb in blood (i.e., venous oxygen saturation $\rightarrow$ 100\%) (Gauthier et al., 2011; Hoge et al., 1999; Krieger et al., 2014). $M$ is estimated with hypercapnia $\left(M_{\mathrm{HC}}\right)$ by measuring changes in CBF and the BOLD signal using

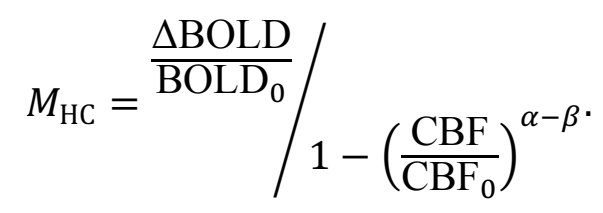


Rather than perturb the oxygen saturation $\left(\mathrm{SO}_{2}\right)$ like a gas-based calibration would, a spin echo image perturbs the spins of the system such that, in the absence of diffusion, the SE will refocus all the spin dephasing induced by deoxyHb present in blood vessels and will, therefore, be equal to the maximum possible GE BOLD signal. To then estimate $R_{2}{ }^{\prime}$, one can acquire another image with $R_{2}{ }^{*}$-weighting, since $R_{2}{ }^{*}=R_{2}+R_{2}{ }^{\prime}$. Acquiring an ASE image is appropriate for this because it will have the same slice profile as the SE image. Example SE and ASE sequences and their transverse signal decays are displayed in Fig. 1. If the spin echo in the ASE image is shifted earlier by a time $\tau$, the signal can be described by

$$
S_{\mathrm{ASE}}(\mathrm{TE}, \tau)=S_{0} \mathrm{e}^{-R_{2} \cdot \mathrm{TE}} \mathrm{e}^{-R_{2}^{\prime} \tau},
$$

where TE is the echo time, $\tau$ is the ASE offset, and $S_{0}$ is the signal at TE $=0$. In the convention used here, $\tau>0$ corresponds to TE occurring a time $\tau$ after the SE occurs. Eq. (3) assumes $\tau>0$ but in the case of $\tau<0, \tau$ should be replaced by $|\tau|$. The SE signal, $S_{\mathrm{SE}}$, is also described by Eq. (3) but with $\tau=0$. If $\tau$ is chosen to be the same as the echo time used for functional imaging, then $M$ from ASE imaging $\left(M_{\mathrm{ASE}}\right)$ can be estimated from the ratio of an SE and ASE image, both acquired at time TE (Blockley et al., 2015):

$$
\begin{aligned}
M_{\mathrm{ASE}} & =\ln \left(S_{\mathrm{SE}} / S_{\mathrm{ASE}}\right) \\
& =R_{2}^{\prime} \tau .
\end{aligned}
$$

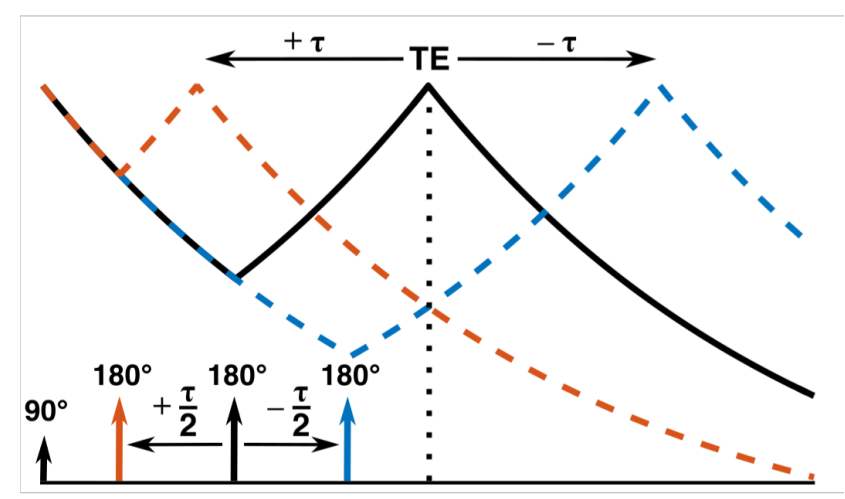

Fig. 1: A schematic of the SE and ASE pulse sequences and their transverse signal decay. All three sequences share the same $90^{\circ}$ excitation pulse and sample the signal at the same echo time (TE, dotted vertical line). The black curve represents the pure SE sequence signal decay. The dashed orange curve represents the ASE sequence signal decay when the ASE offset is $+\tau$. The dashed blue curve represents the ASE sequence signal decay when the ASE offset is $-\tau$. The three signals have no $T_{2}$ decay, no diffusion effects, and only show $R_{2}^{\prime}$-related decay and refocusing. The two ASE signals are equal at TE. 


\subsection{Quadratic Spin Echo Attenuation}

The model of gas-free calibration described above by Eq. (4) applies in the absence of diffusion, where the $180^{\circ}$ pulse will perfectly refocus the dephasing surrounding blood vessels. To determine how SE attenuation from diffusion depends on TE and $\tau$, simulations of the SE and ASE signals were run from vessel networks as a function of vessel radius. These considered the decay resulting from field inhomogeneities only and ignored intrinsic $T_{2}$ decay (details provided in the Methods section below). Fig. 2 shows examples of the simulations with diffusion for three different vessel radii as a function of TE and the case of no diffusion at all. The grey curves show the entire simulated time series, like the simulated signals in Fig. 1, however, there are more spin echo times displayed. The impact of diffusion on the individual simulated time series is that the amount of SE attenuation increases with increasing TE and the time at which maximum refocusing occurs shifts earlier in time than the TE. Also, the amount of decay and refocusing both increase with increasing vessel size. The coloured envelopes represent the signals at the echo times, i.e., when the decays are actually sampled, be it the SE or ASE signals. In the case of no diffusion, the signal refocuses perfectly at the SE times and the SE and ASE signal envelopes are constant across TE. The signal envelopes for all radii and for more values of $\tau$ are shown in Fig. 3. The key qualitative observations are:

1. With respect to $\mathrm{TE}$, the envelopes are all well described by a quadratic-exponential decay early on and by linear-exponential decay later, with the time to transition being proportional to vessel radius and peaking or plateauing for the intermediate-to-larger radii.

2. With respect to $\tau$, the envelope for a given radius is approximately a shifted copy of that radius' envelope from $\tau=0$. The shift is by an amount $\tau$ in time and, by definition, an amount $R_{2}{ }^{\prime} \tau$ along the ordinate. $R_{2}^{\prime}$ is smallest for small vessels (Boxerman et al., 1995), hence the shift is smaller for them and greater for large vessels. 


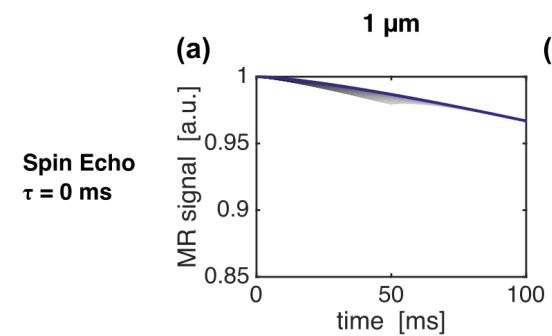

(e)

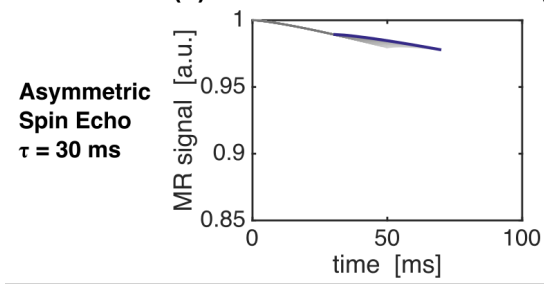

(b)

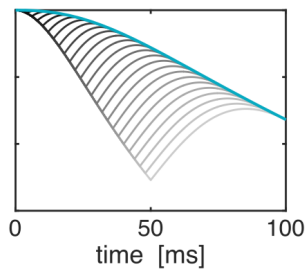

(f)

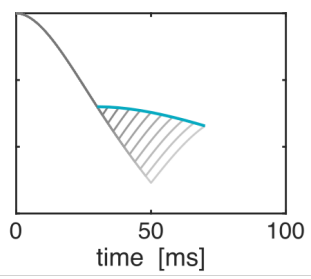

(c)

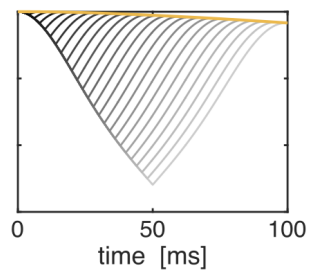

(g)

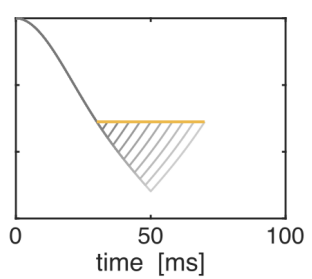

(d)

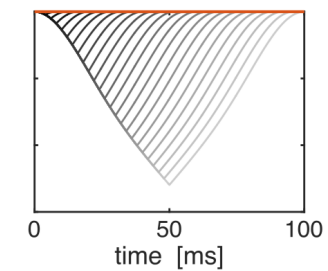

(h)

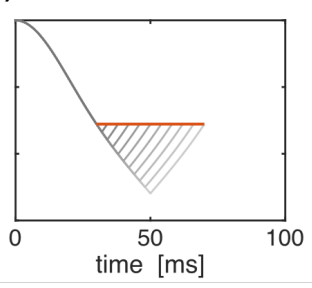

Fig. 2: Example simulated time series from spin echo (top row) and asymmetric spin echo, $\tau=30$ $\mathrm{ms}$, (bottom row) sequences with TE increasing in 4-ms increments. The individual time series for each TE are shown by the greyscale curves, with black to grey representing earlier to later TEs. The coloured signal envelopes represent the signals at the echo times. In the case of the SE simulations, each envelope shows only the signal at the SE times $\left(t_{\mathrm{SE}}\right)$; in the case of the ASE simulations, each envelope shows only the signals at the times $\mathrm{TE}=t_{\mathrm{SE}}+\tau$. The maximum $t_{\mathrm{SE}}$ simulated was $100 \mathrm{~ms}$, therefore, TE ranges from $30 \mathrm{~ms}$ to $70 \mathrm{~ms}$ for the ASE envelope. Each column depicts the simulations from a network containing a different vessel radius $(1 \mu \mathrm{m}, 10 \mu \mathrm{m}$, or $100 \mu \mathrm{m}$ ) or the case of no diffusion. The plotted greyscale ASE signals are for a negative ASE offset but the resulting differences between their signal envelopes and the signal envelopes from the positive offsets were negligible.
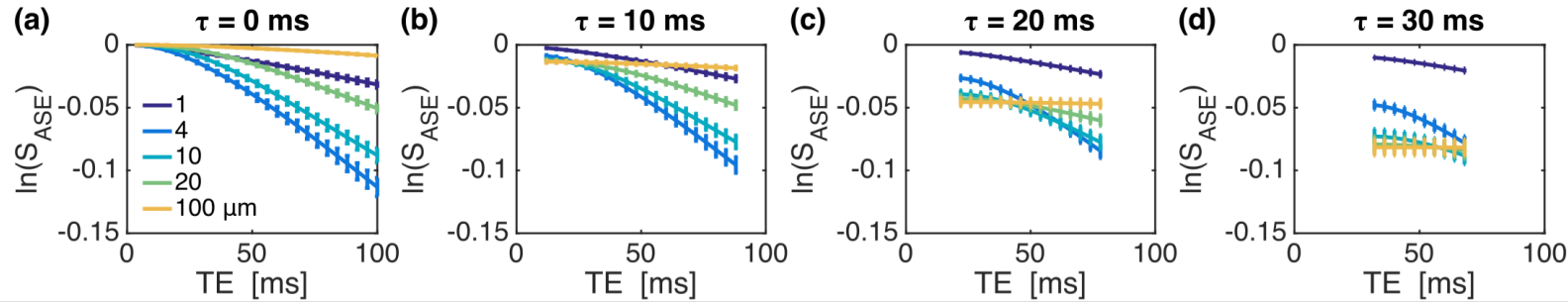

Fig. 3: Logarithm of the ASE signal envelopes as a function of TE and vessel radius. Each subfigure shows the envelope for different ASE offsets, $\tau$, where $\tau=0$ corresponds to the pure spin echo signal. Within each sub-figure, each curve represents the mean signal from a different vessel radius, and they all share the legend in (a). The ranges of TE for each sub-figure are $\tau \leq \mathrm{TE} \leq 100$ $\mathrm{ms}-\tau$. Error bars represent the mean $\pm \mathrm{SD}$ of the simulations across networks.

These observations, including the quadratic-to-linear transition times, are examined in greater detail in the Supplementary Material. The shift along the ordinate is $R_{2}{ }^{\prime} \tau$, by definition, 
because at the beginning of each curve, where $\mathrm{TE}=\tau$, the signal is a pure gradient echo signal, implying that no refocusing pulse has been applied and that the signal decay is due solely to the intrinsic $R_{2}{ }^{\prime}$ of the simulation network. If the gradient echo decay is monoexponential, the signal at $\mathrm{TE}=\tau$ will therefore be reduced by a factor $\exp \left(R_{2}^{\prime} \tau\right)$. However, this decay is not monoexponential, even in the absence of diffusion (Yablonskiy and Haacke, 1994), so $R_{2}{ }^{\prime}$ will be the apparent $R_{2}{ }^{\prime}$ for the given $\tau$.

The simulations above were repeated on networks populated by two different distributions of vessel radii, referred to here as the Lauwers (Lauwers et al., 2008) and Frechet (Germuska et al., 2013) distributions, and shown in Fig. 4. The Lauwers distribution had very few radii $>10 \mu \mathrm{m}$, whereas the Frechet distribution peaked near $10 \mu \mathrm{m}$ and had a long tail out to $60 \mu \mathrm{m}$. Similar signal behaviour can be seen in the simulations from the Lauwers and Frechet vessel-size distributions, shown in Fig. 5. In this case, the Lauwers distribution, which is primarily microvascular, appears to transition from quadratic- to linear-exponential decay earlier than the Frechet distribution and its vertical shift is smaller, consistent with the results in Fig. 3.

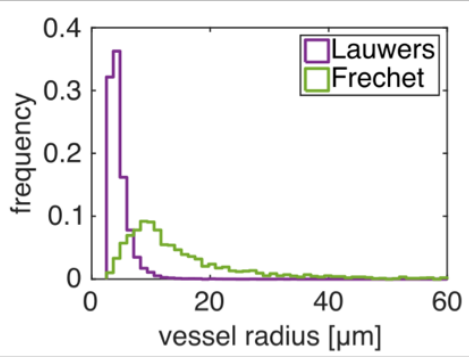

Fig. 4: Histograms of the Lauwers and Frechet vessel-size distributions. The values represent the frequency with which the given ranges of radii occur across all vessel networks of a given distribution. 

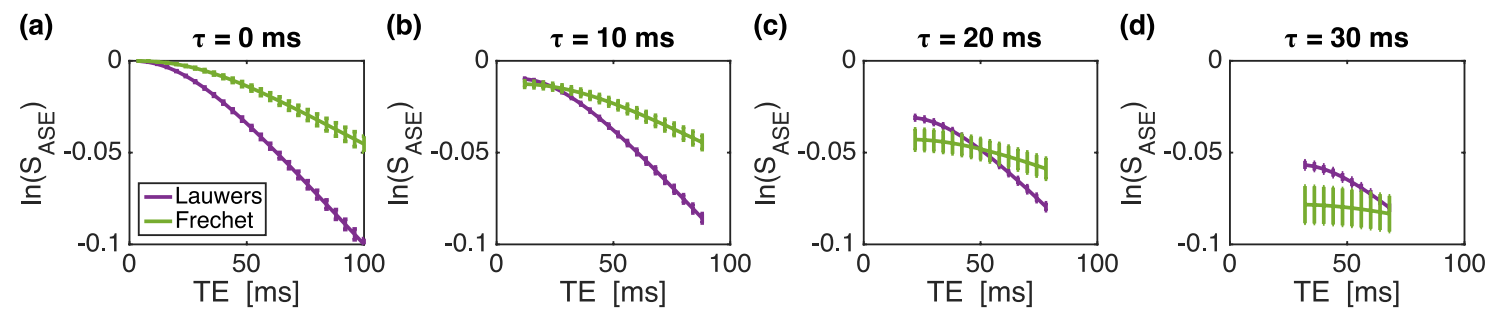

Fig. 5: Logarithm of the ASE signal envelopes as a function of TE for the Lauwers and Frechet vessel-size distributions. Each sub-figure shows the envelope for different ASE offsets, $\tau$, where $\tau$ $=0$ corresponds to the pure spin echo signal. All sub-figures share the legend in (a). Error bars represent the mean $\pm \mathrm{SD}$ of the simulations across networks.

We propose that the diffusion-induced SE attenuation can be characterized by ignoring the transition to linear-exponential decay and considering the attenuation as an additional quadratic-exponential decay term with rate constant $\left(R_{2, \text { diff }}\right)^{2}$. This decay would commence at $\mathrm{TE}=\tau$ and the ASE signal in Eq. (3) would be modified as

$$
S_{\mathrm{ASE}}(\mathrm{TE}, \tau)=S_{0} e^{-R_{2} \cdot \mathrm{TE}} e^{-R_{2}^{\prime} \cdot \tau} e^{-\left(R_{2, d i f f}\right)^{2}(\mathrm{TE}-\tau)^{2}} .
$$

We refer to this model of the ASE signal as the quadratic ASE (q-ASE) model. The $\left(R_{2, \text { diff }}\right)^{2}$ term can be visualized by plotting the expression

$$
\begin{aligned}
\Delta R_{2}(\mathrm{TE}) & =-\frac{\ln S_{\mathrm{SE}}}{\mathrm{TE}}=-\frac{\ln \left(S_{A S E}(\tau=0)\right)}{\mathrm{TE}} \\
& =-\ln S_{0} / \mathrm{TE}+R_{2}+\left(R_{2, \text { diff }}\right)^{2} \mathrm{TE} \\
& =\left(R_{2, \text { diff }}\right)^{2} \mathrm{TE} .
\end{aligned}
$$

The final equality is a simplification for the simulations only because $S_{0}$ is normalized to 1 and the intrinsic $R_{2}$ is set to 0 . This indicates that $\left(R_{2, \text { diff }}\right)^{2}$ can be estimated from the initial slope of $\Delta R_{2}$ vs. TE.

Taking the ratio $\ln \left(S_{\mathrm{SE}} / S_{\mathrm{ASE}}\right)$ using Eq. (5) gives

$$
\begin{aligned}
\ln \left(\frac{S_{\mathrm{SE}}}{S_{\mathrm{ASE}}}\right) & =\ln \left(\frac{S_{\mathrm{ASE}}(\tau=0)}{S_{\mathrm{ASE}}(\tau)}\right) \\
& =R_{2}^{\prime} \tau+\left(R_{2, \text { diff }}\right)^{2} \tau^{2}-2\left(R_{2, \text { diff }}\right)^{2} \tau \mathrm{TE} .
\end{aligned}
$$


Since $\tau \leq \mathrm{TE}$, the ratio will always be less than $R_{2}^{\prime} \tau$ and is consistent with $M$ being underestimated from $\ln \left(S_{\mathrm{SE}} / S_{\mathrm{ASE}}\right)$ alone, as in Eq. (4). From the linear TE dependence, it should be possible to estimate $\left(R_{2, d i f f}\right)^{2}$ and $R_{2}^{\prime}$ if this ratio is measured at two or more echo times. $M$ can then be estimated using

$$
M=e^{R_{2}^{\prime} \cdot \mathrm{TE}_{f u n c}}-1 \simeq R_{2}^{\prime} \mathrm{TE}_{f u n c},
$$

where $\mathrm{TE}_{\text {func }}$ is now the BOLD echo time of the functional experiment that the calibration is to be applied to.

Alternatively, one could use a fixed TE and fit the quadratic dependence of Eq. (7) using several values of $\tau$. However, more data would be required to fit the quadratic relationship and other experiments and theories of SE decay have shown that this ratio can become quadratic around the SE as a function of TE or $\tau$ in the absence of diffusion (An and Lin, 2003; Yablonskiy, 1998; Yablonskiy and Haacke, 1994). Therefore, these two distinct quadratic behaviours could confound the estimate of $\left(R_{2, \text { diff }}\right)^{2}$. If one tries to avoid the quadratic ASE attenuation regime altogether by sampling at long $\tau$ and TE, when the attenuation has transitioned to linear-exponential decay, then the $R_{2}{ }^{\prime}$ underestimation will persist and it will be irrecoverable. This can be shown by replacing $\exp \left[-\left(R_{2, d i f f}\right)^{2}(\mathrm{TE}-\tau)^{2}\right]$ in Eq. (5) with a linearexponential decay equivalent and then calculating the log-ratio. In this case, the linear decay term can describe the underestimation of $R_{2}{ }^{\prime}$ but it becomes indistinguishable from $R_{2}{ }^{\prime}$ itself as they have the same dependence on $\tau$. Therefore, sampling in the linear decay regime provides no straightforward means for correcting the SE attenuation.

\section{Methods}

\subsection{Simulations}

The simulations in this study used the deterministic diffusion method (Bandettini and Wong, 1995). This consisted of populating a two-dimensional (2D) plane with vessels to the desired CBV, where blood vessels were modelled as cylinders perpendicular to the plane. To model the 
random orientation of the vessels, the direction of the main $B_{0}$ field was randomized for each vessel (Miller and Jezzard, 2008). This vessel-wise randomization of the $B_{0}$ direction was used rather than physically reorienting the vessels relative to the plane because it resulted in a more easily controlled CBV and still generated the desired distribution of field offsets since the field offsets along a direction parallel to a cylinder are invariant. An example vessel distribution and the resulting field offsets are shown in Fig. 6.
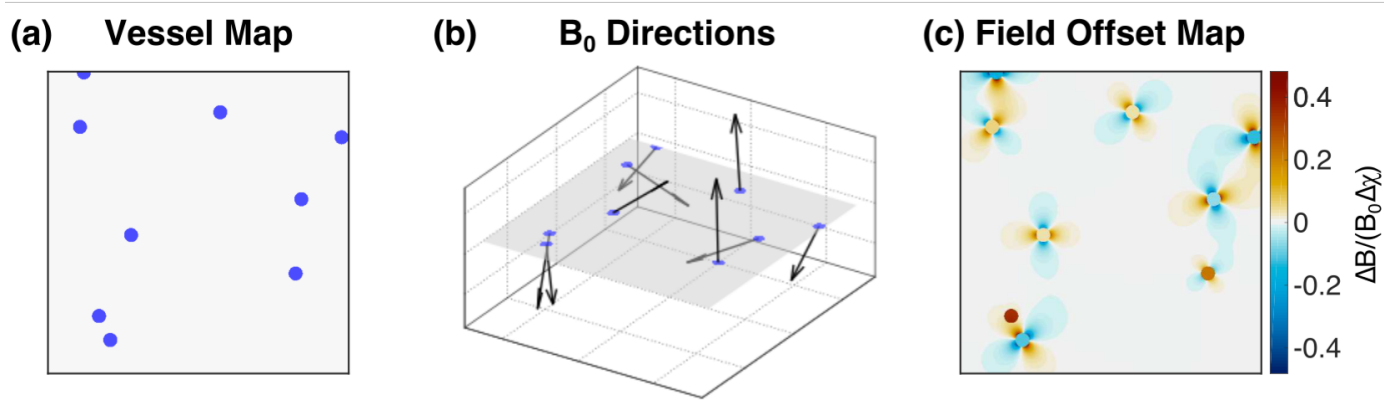

Fig. 6: (a) Example 2D vessel map. The blue circles correspond to the vessel cross sections. (b) The random $B_{0}$ directions assigned to each vessel. The directions are represented by the arrows and are overlaid on a semi-transparent version of the vessel map. (c) The field offset map generated by the vessels. All the vessels were assigned the same susceptibility offset, $\Delta \chi$, and the map was normalized by $B_{0} \Delta \chi$.

The deterministic diffusion simulation method spatially discretizes the area over which the simulation is run onto a lattice and, for a given distribution of vessels, calculates the field offsets generated by them over this lattice (Bandettini and Wong, 1995; Pannetier et al., 2013). Following an initial $90^{\circ}$ excitation pulse, each element of the lattice has a uniform magnetization with an initial phase of 0 and magnitude of 1 . In time steps, $\delta t$, the magnetization at the $(k, l)$-th lattice element, $M_{k l}$, precesses by an angle $\Delta \phi_{k l}=\gamma \Delta B_{k l} \delta t$, where $\Delta B_{k l}$ is the field offset at that lattice element. Diffusion is modelled by an isotropic, Gaussian blurring of the magnetization along each dimension independently. This was implemented by linear convolution of the magnetization with the 1D discrete diffusion kernel with a width parameter equal to the expected mean-square displacement of the spins, $\sigma^{2}=2 D \delta t$, where $D$ is the diffusion coefficient of water in tissue (Pannetier et al., 2014). The $k$-th element of the kernel is given by 


$$
D_{k}=e^{-(\sigma / \Delta x)^{2}} I_{k}\left((\sigma / \Delta x)^{2}\right),
$$

where $\Delta x$ is the physical spacing between lattice elements and $I_{k}$ is the modified Bessel function of the first kind of order $k$. The half-width of the kernel was a minimum of $6 \sigma$ and was extended, if necessary, until $1-\Sigma D_{k} \leq 5 \mathrm{e}-8$. This requirement ensures that the kernel is normalized and it was empirically tested for accuracy with preliminary simulation tests. $180^{\circ}$ refocusing pulses were modelled by taking the complex conjugate of the magnetization at each lattice element. Finally, the signal magnitude at the $n$-th time point is given by

$$
S_{n}=\frac{1}{N^{2}}\left|\sum_{k, l} M_{k l, n}\right|,
$$

where $N$ is the number of lattice elements summed along each dimension and the sum was only performed over the central $1 / 3$ of the lattice width along each dimension to avoid convolution edge effects.

The field offsets generated by each vessel were given by modelling them as infinite cylinders perpendicular to the simulation plane with (Ogawa et al., 1993):

$$
\Delta B_{0}=\left\{\begin{array}{ll}
\frac{1}{2} \Delta \chi B_{0}\left(\frac{R}{r}\right)^{2} \sin ^{2}(\theta) \cos (2 \phi) & r \geq R \\
\frac{1}{6} \Delta \chi B_{0}\left(3 \cos ^{2}(\theta)-1\right) & r<R
\end{array},\right.
$$

where $R$ is the vessel radius, $r$ is the distance from the point of interest to the centre of the vessel, $\theta$ is the angle between $B_{0}$ and the centre line of the vessel, $\phi$ is the angle between the vector defined by $r$ and the component of $B_{0}$ in the plane, and $\Delta \chi$ is the susceptibility difference between the blood within the vessel and the surrounding tissue. The field offsets from each vessel were independently calculated and summed across the lattice to give $\Delta B_{k l}$.

To determine the echo time dependence of the SE attenuation, simulations were run from time 0 (i.e., immediately after excitation) up to the spin echo time $\left(t_{\mathrm{SE}}\right)$, with $t_{\mathrm{SE}}$ incremented in 2-ms steps from 2 to $100 \mathrm{~ms}$. Since the simulations only ran up to $t_{\mathrm{SE}}$, the ASE signals were determined using negative ASE offsets (i.e., $\tau<0$ ) and testing showed that the signal difference 
for positive vs. negative $\tau$ was negligible (results not shown). Simulations were performed using $B_{0}=3 \mathrm{~T}, \mathrm{CBV}=2 \%$ (representing venous $\mathrm{CBV}$ ), $\Delta \chi=4 \pi \cdot 0.04 \mathrm{ppm}$, diffusion coefficient $D=$ $0.8 \mu \mathrm{m}^{2} / \mathrm{ms}$, and time step $\delta t=0.25 \mathrm{~ms}$. This $\Delta \chi$ approximately corresponds to vessels with an $\mathrm{SO}_{2}$ of $60 \%$ and hematocrit of $40 \%$ and where tissue has the same susceptibility as fully oxygenated blood (Berman et al., 2016; Spees et al., 2001). Additional CBVs and $\Delta \chi$ s were examined in the Supplementary Material. Ten networks were randomly seeded with 1.0- $\mu \mathrm{m}$ vessels on a $1020^{2}$ lattice with a side length of $255 \mu \mathrm{m}$ isotropic. At this resolution, each vessel could be sampled up to eight times across its diameter, and using higher resolutions had a negligible impact on the results. These networks were reused to perform the simulations for radii up to $20 \mu \mathrm{m}$ by assigning them an effective lattice size of $255 \mu \mathrm{m} \times R / 1.0 \mu \mathrm{m}$. For $R \geq 20 \mu \mathrm{m}$, the same scaling was employed, however, the lattice was resampled to a $6375^{2}$ grid to ensure the diffusion kernel and field offset map were sampled at a high enough spatial resolution. Only a single diffusion coefficient was used throughout since it is not the diffusion coefficient alone that impacts signal evolution, it is diffusion time, defined by the ratio of the squared vessel radius and the diffusion coefficient $\left(R^{2} / D\right)$. Since vessel radii were varied from 1 to $100 \mu \mathrm{m}$, the effect of diffusion variation was indirectly probed over a wide range. All simulations were run using MATLAB R2015a (MathWorks, Natick, MA).

For the vessel-size distributions, the Lauwers distribution was obtained using the parameters for the complete vessel network in (Lauwers et al., 2008) with the radii ranging from 3.0-40 $\mu \mathrm{m}$. The Frechet distribution was implemented in MATLAB using the gevrnd function with the input parameters $\mu=10.1 \mu \mathrm{m}, \sigma=5.8$, and $k=0.41$ taken from (Germuska et al., 2013) and with the radii ranging from $2.5-60 \mu \mathrm{m}$.

To evaluate the hypothesis that $\ln \left(S_{\mathrm{SE}} / S_{\mathrm{ASE}}\right)$ may have a linear dependence on TE, the ratio was calculated from the previous simulations as a function of TE and $\tau$. The calibration constant, $M$, was then calculated from simulations with a finer division of radii from 1-100 $\mu \mathrm{m}$ and the Lauwers and Frechet radii distributions using two TEs to fit for $R_{2}{ }^{\prime}$ and $\left(R_{2, \text { diff }}\right)^{2}$ with Eq. (7). This was tested with $\tau=+30 \mathrm{~ms}$ and TEs of $40 \mathrm{~ms}$ and $50 \mathrm{~ms}$. The fitted $R_{2}{ }^{\prime}$ values from the two different $\tau$ values were substituted into Eq. (8) to calculate $M$ using $\mathrm{TE}_{\text {func }}=30 \mathrm{~ms}$. For comparison, $M$ was also calculated at a single TE of $40 \mathrm{~ms}$ with $\tau=+30 \mathrm{~ms}$ using Eq. (4), as in 
Blockley et al. (2015). These ASE-based $M$ calculations were compared against the "ideal" $M$ given by the maximum possible gradient echo percent signal change at $\mathrm{TE}=30 \mathrm{~ms}$. Since these simulations ignored $T_{2}$ relaxation, the maximum signal was taken to be 1 .

\subsubsection{Intrinsic $T_{2}$ Decay}

To estimate the differential influence of intravascular and extravascular $T_{2}$ relaxation, the simulated signals $\left(S_{\text {sim }}\right)$ from the Lauwers and Frechet distributions were combined with a model for intravascular signal decay $\left(S_{\mathrm{IV}}\right)$, giving the net tissue signal,

$$
\begin{gathered}
S_{n e t}(\mathrm{TE}, \tau)=(1-\mathrm{CBV}) S_{s i m}(\mathrm{TE}, \tau) \exp \left(-\mathrm{TE} / T_{2, t}\right) \\
+\mathrm{CBV} S_{\mathrm{IV}}(\mathrm{TE}, \tau)
\end{gathered}
$$

where $T_{2, t}=110 \mathrm{~ms}$ was used for grey matter(Wansapura et al., 1999). $S_{\text {IV }}$ is an analytical expression that describes the complete transverse decay and refocusing of an ASE or SE sequence from a system in the motional narrowing regime, such as blood (Berman and Pike, 2017):

$$
\begin{gathered}
S_{\mathrm{IV}}(\mathrm{TE}, \tau)=\exp \left\{-\frac{\gamma^{2}}{2} G_{0} \tau_{D}^{2}\right. \\
\times\left[\frac{\mathrm{TE}}{\tau_{D}}+\left(\frac{1}{4}+\frac{\mathrm{TE}}{\tau_{D}}\right)^{\frac{1}{2}}+\frac{3}{2}\right. \\
-2\left(\frac{1}{4}+\frac{\mathrm{TE}-(\mathrm{TE}-\tau) / 2}{\tau_{D}}\right)^{\frac{1}{2}} \\
\left.\left.-2\left(\frac{1}{4}+\frac{(\mathrm{TE}-\tau) / 2}{\tau_{D}}\right)^{\frac{1}{2}}\right]\right\} \exp \left(-\mathrm{TE} / T_{2, b \mid 0}\right),
\end{gathered}
$$

where $G_{0}$ is the mean square field inhomogeneity, $T_{2, b \mid 0}=189 \mathrm{~ms}$ is the intrinsic $T_{2}$ of blood, and $\tau_{D}=r_{c}^{2} / D$ with $r_{c}=2.6 \mu \mathrm{m}$, the characteristic size of red blood cells, and $D=2.0 \mu \mathrm{m}^{2} / \mathrm{ms}$ is the diffusion coefficient of blood. $T_{2, b \mid 0}$ and $r_{c}$ were obtained from fits to experimental blood relaxometry data (Berman and Pike, 2017; Chen and Pike, 2009) and $G_{0}$ is given by (Jensen and Chandra, 2000; Sukstanskii and Yablonskiy, 2003) 


$$
G_{0}=\frac{4}{45} \operatorname{Hct}(1-\mathrm{Hct})\left(4 \pi \cdot 0.27 \mathrm{ppm} \cdot\left(0.95-\mathrm{SO}_{2}\right) B_{0}\right)^{2}
$$

Hct is the hematocrit of blood and was set to $40 \%, \mathrm{SO}_{2}$ was set to $60 \%$. The maximum signal in this case is given by $S_{\text {net, } \max }=(1-\mathrm{CBV}) \exp \left(-\mathrm{TE} / T_{2, t}\right)+\mathrm{CBV} \exp \left(-\mathrm{TE} / T_{2, b \mid 0}\right)$, corresponding to $\mathrm{SO}_{2}=95 \%$ (Spees et al., 2001). Rather than use just two echo times for the qASE model, these comparisons used TEs of 40,50, 60, and $70 \mathrm{~ms}$, to be consistent with the in vivo acquisition.

Random noise was also added to these simulations to examine the impact of the signal-tonoise ratio (SNR) on the estimates of $\left(R_{2, d i f f}\right)^{2}$ and $M$. These results are described in the Supplementary Material.

\subsection{In Vivo Study}

\subsubsection{MR Imaging}

We evaluated the quadratic ASE model on nine healthy participants (4 female, 5 male; 19-39 years of age; mean age $=28 \pm 7$ years) using a $3 \mathrm{~T}$ scanner (Discovery 750, GE Healthcare, Waukesha, WI). The study was approved by our institutional review board and all participants gave informed written consent. Subjects were scanned with a 2D ASE EPI sequence with FLAIR preparation. Imaging parameters included: field of view $=224 \times 224 \mathrm{~mm}^{2} ; 96 \times 96$ matrix;

bandwidth $=250 \mathrm{kHz}$; slice thickness $=2.0 \mathrm{~mm}+1.0-\mathrm{mm}$ gap; 34 slices (interleaved); flip angle $=90^{\circ}$; ASSET factor $=2$; four TEs of 42, 50, 60, and $70 \mathrm{~ms} ; \tau=0$ and $30 \mathrm{~ms}$; inversion time $(\mathrm{TI}) /$ repetition time $(\mathrm{TR})=2.0 / 8.0 \mathrm{~s}$. This combination of TI/TR was determined to null the signal from CSF in preliminary testing on two additional subjects. Slices were angled parallel to the anterior commissure-posterior commissure line and aligned to the top of the brain, typically resulting in whole-cerebrum coverage and partial cerebellum coverage. For each TE- $\tau$ image, 15 complex volumes were acquired with two additional dummy volumes.

During the hypercapnic calibration, BOLD and CBF changes were assessed with a dualecho pseudo-continuous arterial spin labelling (ASL) sequence with a 2D EPI readout and the 
following imaging parameters: field of view $=224 \times 224 \mathrm{~mm}^{2} ; 64 \times 64$ matrix; bandwidth $=250$ $\mathrm{kHz}$; slice thickness $=5.0 \mathrm{~mm}+1.0$-mm gap; 18 axial slices acquired in a top-down order; flip angle $=90^{\circ} ;$ ASSET factor $=1.5 ; \mathrm{TE}_{1} / \mathrm{TE}_{2}=9.5 / 30 \mathrm{~ms} ; \mathrm{TR}=3.6 \mathrm{~s} ;$ ASL label duration $=1600$ $\mathrm{ms}$; post-label delay $=900 \mathrm{~ms}$. Slices were aligned to the top of the brain and the labelling plane was located $20 \mathrm{~mm}$ inferior of the most inferior slice. 100 volumes were acquired with four additional dummy volumes.

A separate $B_{0}$ field map was acquired using a 2D, fast spoiled gradient recalled echo, three-echo sequence with a monopolar readout, a field of view matched to the ASE images, $256 \times 256$ matrix, $\mathrm{TE}_{1}=4.45 \mathrm{~ms}$, echo spacing $=2.1 \mathrm{~ms}, \mathrm{TR}=500 \mathrm{~ms}$, and flip angle $=30^{\circ}$. High resolution structural images were acquired using a 3D MP-RAGE acquisition (Mugler and Brookeman, 1990) with a 1 -mm isotropic resolution, $192 \times 256 \times 256$ matrix, TR/TI/TE $=$ $6.66 / 650 / 2.93 \mathrm{~ms}$, and $10^{\circ}$ flip angle.

\subsubsection{Hypercapnia Gas Challenge}

All subjects underwent a hypercapnic gas challenge in the MR scanner consisting of breathing medical air for $2 \mathrm{~min}, 5 \% \mathrm{CO}_{2}$ in medical air for $2 \mathrm{~min}$, then medical air for $2 \mathrm{~min}$. Participants were delivered the gases with an automated flow controller (FloBox 954, Sierra Instruments, Inc., L Monterey, CA) connected to a non-rebreathing circuit with an extended expired gas reservoir that was open to the room at its end (Tancredi et al., 2014). Gases were delivered at a constant flow rate of $20 \mathrm{l} / \mathrm{min}$, and partial pressures of oxygen and carbon dioxide in the breathing mask were monitored with BIOPAC $\mathrm{O}_{2} 100 \mathrm{C}$ and $\mathrm{CO}_{2} 100 \mathrm{C}$ modules connected to a BIOPAC MP150 acquisition unit (BIOPAC Systems, Inc., Goleta, CA). The mean end-tidal partial pressures of $\mathrm{CO}_{2}\left(\mathrm{P}_{\mathrm{ETCO}}\right)$ and $\mathrm{O}_{2}\left(\mathrm{P}_{\mathrm{ET}} \mathrm{O}_{2}\right)$ at rest were determined from the $60 \mathrm{~s}$ of endtidal measurements prior to the hypercapnia challenge, and the mean changes in the end-tidal values were determined from the final $60 \mathrm{~s}$ of the hypercapnia challenge.

\subsubsection{Image Processing}


All image analysis was ultimately performed in the individual subjects' ASL image space, however, image preprocessing was generally performed in each image's native space. A combination of tools from Statistical Parametric Mapping (SPM) 8 (Wellcome Trust Centre for Neuroimaging, London, UK), FMRIB Software Library (FSL) v5.0.7 (Jenkinson et al., 2012), and MATLAB were used for image analysis.

For each TE- $\tau$ ASE combination, the image volumes were motion corrected using SPM realign, complex-averaged across time, then converted to magnitude images. The complex averaging preserved the noise properties of the lower signal-to-noise ratio regions (Gudbjartsson and Patz, 1995). The mean magnitude images were coregistered to the mean TE $/ \tau=42 / 0 \mathrm{~ms}$ image using SPM coreg and then brain extracted using FSL BET (Smith, 2002). The TE $/ \tau=42 / 0$ ms brain extracted, distortion corrected image is referred to as the ASE reference image. Geometric distortion correction of the images was performed using the $B_{0}$ field map with FSL FUGUE (Jezzard and Balaban, 1995). The field map was fit by nonlinear estimation in the complex domain (Liu et al., 2013) and smoothed by fitting it to a 3D smoothing spline in MATLAB. Voxels in the ASE images where the warp was greater than 1 voxel were excluded from later analyses.

The ASL images were motion corrected using the SPM ASL toolbox (Wang, 2012). Simultaneous geometric distortion correction and registration of the mean echo 1 image to the structural image was performed using boundary-based registration with FSL's epi_reg utility (white matter (WM) segmentation described below) (Greve and Fischl, 2009; Jenkinson and Smith, 2001). The distortion correction was then applied to all echo 1 and echo 2 images.

Calculation of the $M$ values was performed on anatomically defined grey matter regions of interest (ROIs). Segmentation of the structural image was performed using SPM8 new segment (Ashburner and Friston, 2005), giving tissue probability maps and the nonlinear deformation into MNI space. The inverse deformation fields were used to transform four atlasbased ROIs corresponding to the frontal, occipital, parietal, and temporal lobes from MNI space to subject space (Mazziotta et al., 2001). These ROIs were then multiplied with the GM tissue probability maps, transformed to ASL-space, and thresholded at 0.75 to produce binary masks. A 
fifth ROI consisting of all GM thresholded at 0.75 was also used. The transformation matrices to the structural image for the ASE reference image were estimated using epi_reg: given the lack of GM-WM contrast and the pronounced CSF-parenchyma contrast in the FLAIR-ASE images, the CSF segmentation was used for the contrast boundary.

Macroscopic field inhomogeneities unequally affect the ASE and SE signal intensities and, hence, the apparent $R_{2}^{\prime}$; therefore, several efforts were made to exclude regions of excess signal intensity distortions arising from field inhomogeneities and to compensate for moderate signal dropout. The field gradients across the slice, frequency, and phase encode directions were calculated numerically by central differences on the smoothed field map. The $\tau=30 \mathrm{~ms} \mathrm{ASE}$ image intensities were corrected for gradients across the slice, $G_{s}$, by dividing them on a voxelwise basis by the factor $\operatorname{sinc}\left(\gamma G_{s} \tau \Delta z / 2 \pi\right)$ (Yablonskiy, 1998), where $\gamma=2.675 \times 10^{8} \mathrm{rad} / \mathrm{s} / \mathrm{T}$ is the gyromagnetic ratio of ${ }^{1} \mathrm{H}$, and $\Delta z$ is the slice thickness. The sinc correction method relies on an ideal square slice profile; this assumption was tested using Bloch simulations of the pulse sequence and by measurement of the slice profile in a phantom and it was found to be in close agreement up to the first zero of the sinc function. Voxels where the sinc term was less than 0.5 were excluded from the ROIs for analysis. In-plane field gradients can lead to gradual dephasing and, for gradients above a critical threshold, can push the gradient echoes in the EPI readout trajectory out of the $k$-space acquisition window, resulting in abrupt signal loss (Deichmann et al., 2002; Weiskopf et al., 2007). Therefore, only voxels where the in-plane gradient magnitude was less than $50 \%$ of the critical gradient magnitude were included for analysis. This corresponded to including voxels where the gradient magnitude was less than $84 \mu \mathrm{T} \mathrm{m}^{-1}$ along the readout direction and less than $56 \mu \mathrm{T} \mathrm{m}^{-1}$ along the phase encode direction. Despite these precautions, the estimated $R_{2}{ }^{\prime}$ values in the temporal lobe were still artefactually elevated; therefore, only slices superior to the cerebellum were included in the ROIs for analysis.

\subsubsection{Data Analysis}

After transformation of all ASE images into each subject's own ASL space, their intensities were averaged across the five ROIs and the ratio of the mean SE over the mean ASE signal was calculated at each echo time. Non-linear fitting of this ratio vs. TE to the q-ASE model in Eq. (7) 
was performed in MATLAB using lsqcurvefit with the trust-region-reflective algorithm. This resulted in fits for $R_{2}{ }^{\prime}$ and $\left(R_{2, d i f f}\right)^{2}$ from which $M$ was then determined using the non-linearized version of Eq. (8). Whether the q-ASE model statistically significantly increased the $M$ values relative to those determined using only the $\mathrm{TE}=42 \mathrm{~ms}$ data was assessed using a single-sided Wilcoxon signed rank test for each ROI, with $P<0.05$ deemed significant.

For comparison, $M$ was calculated across the ROIs from the dual-echo ASL images during the hypercapnia challenge. To calculate CBF-weighted changes, tag-control subtraction of the first echo images was performed using sinc interpolation. The second echo images were used as BOLD-weighted images. The percent signal changes of the BOLD and CBF signals were determined from their modelled responses using a general linear model analysis on the averaged signals across the ROIs (Bulte et al., 2012). Both the averaged BOLD and CBF signal time courses were modelled by convolving the hypercapnia stimulus paradigm with a gamma-variate function with a mean lag of $30 \mathrm{~s}$ and a standard deviation of $15 \mathrm{~s}$ (Mazerolle et al., 2016). The temporal derivative was included as an additional regressor to account for temporal delays in the responses. In the BOLD images only, linear drift and tag-control nuisance regressors were included. $M$ values for each ROI were then calculated using Eq. (2) with $\alpha=0.2$ (Chen and Pike, 2010b) and $\beta=1.3$ (Bulte et al., 2009; Uludag et al., 2004). Whether the hypercapnic $M$ values differed significantly from the ASE and q-ASE $M$ values was determined using a two-sided Wilcoxon signed rank test for each ROI, with $P<0.05$ deemed significant.

\section{Results}

\subsection{Simulations}

The quadratic decay term, $\left(R_{2, \text { diff }}\right)^{2}$, is visualized in Fig. 7 by plotting the initial slope of $\Delta R_{2}$ vs. TE for the SE signals. The fitted slopes, which give $\left(R_{2, d i f f}\right)^{2}$ by Eq. (6), appear to follow a sigmoidal relationship that is monotonically decreasing with vessel radius, as shown in Fig. $7 \mathrm{~b}$. The $\left(R_{2, \text { diff }}\right)^{2}$ values for the Lauwers and Frechet vessel radius distributions are $(14.1 \pm 0.5) \mathrm{s}^{-2}$ and $(5.3 \pm 0.6) \mathrm{s}^{-2}$, respectively. From Fig. $7 \mathrm{a}$ and $\mathrm{c}$, if $\left(R_{2, \text { diff }}\right)^{2}$ was estimated by fitting the 
tangent at later times, there would be an underestimation that would be worse for the smaller radii.
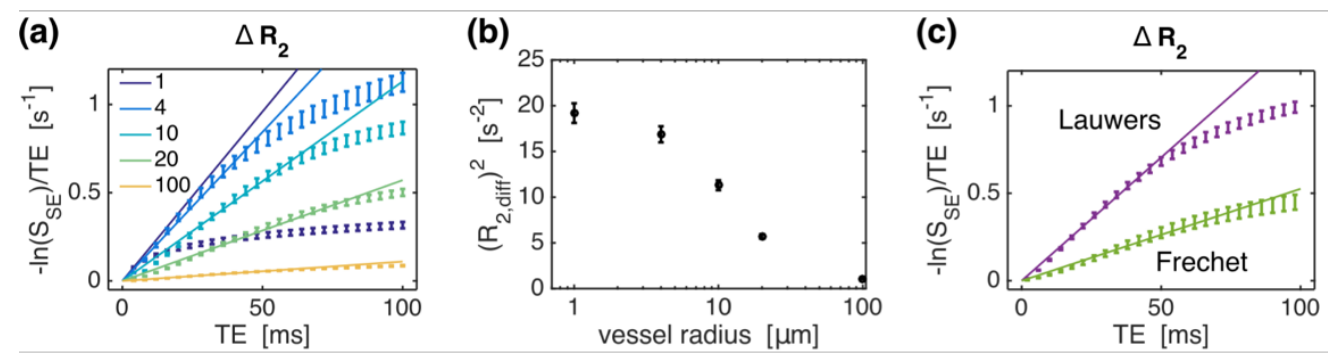

Fig. 7: The TE dependence of $\Delta R_{2}$ for individual radii (in $\mu \mathrm{m}$ ) (a) and for the two distributions of radii (c). Error bars in (a) and (c) show the mean $\pm \mathrm{SD}$ of the simulated $\Delta R_{2}$ values. The initial relationships of $\Delta R_{2}$ vs. TE are plotted with the straight lines. The slopes of the lines reflect the $\left(R_{2, \text { diff }}\right)^{2}$ term and those values from (a) are plotted as a function of vessel radius in (b). The slopes in (c) for the Lauwers and Frechet distributions are $(14.1 \pm 0.5) \mathrm{s}^{-2}$ and $(5.3 \pm 0.6) \mathrm{s}^{-2}$, respectively.

The $\log$-ratio, $\ln \left(S_{\mathrm{SE}} / S_{\mathrm{ASE}}\right)$, as a function of TE is shown in Fig. 8 for a subset of $\tau$ offsets. For a given TE and given $\tau$, the curves for each radius are vertically offset from each other and they increase monotonically with vessel size. This reflects the true difference in $R_{2}{ }^{\prime}$ for the different vessel sizes that is widely known for GE BOLD (Boxerman et al., 1995). At the vesselsize extremes of $1 \mu \mathrm{m}$ and $100 \mu \mathrm{m}$, both ratios are relatively flat as a function of TE. These arise from two different mechanisms, however. The ratio is flat for the $1-\mu \mathrm{m}$ simulations because the signal rapidly transitioned from quadratic to linear decay, as observed in Fig. 3. Conversely, the ratio is relatively flat for the $100-\mu \mathrm{m}$ simulations because the diffusion-induced SE attenuation is small since the scale of diffusion is much less than that of the field offsets surrounding the vessels. In between these radii, it can be seen how the curves transition. For the shortest $\tau$ offset, the slopes monotonically decrease with increasing radius (ignoring the $1 \mu \mathrm{m}$ simulations). At the later $\tau$ offsets, the slopes no longer decrease monotonically with increasing radius but rather they peak around $10 \mu \mathrm{m}$. This change in behaviour with increasing $\tau$ is the result of the early quadratic decay being missed in the SE signals for the smaller vessel sizes as TE is increased, as predicted above by Fig. 7. 

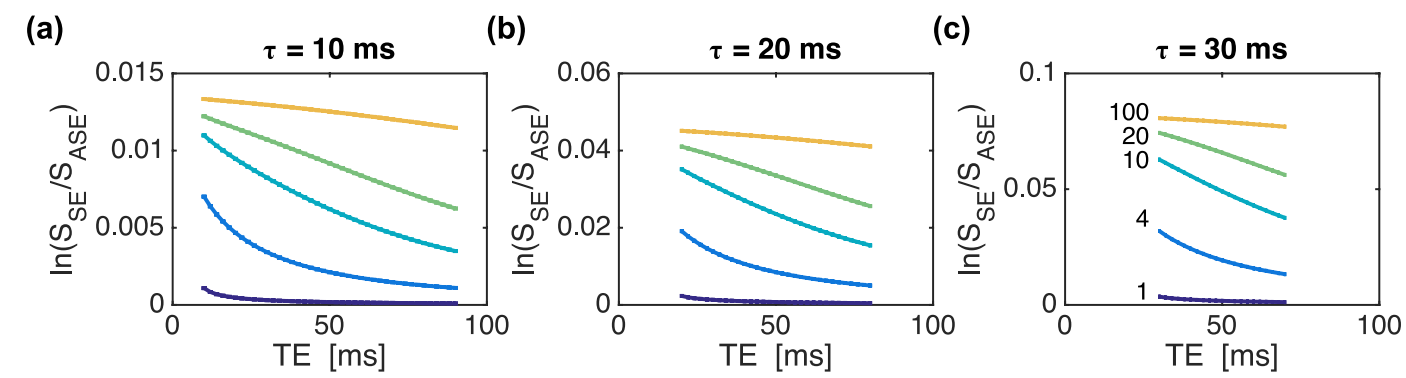

Fig. 8: The log-ratio of the SE signal $(\tau=0 \mathrm{~ms})$ over the ASE signal for three different ASE offsets, as a function of TE and vessel radius. The radii, in $\mu \mathrm{m}$, are labelled in (c). Error bars represent the mean \pm the standard deviation of the log-ratio across all simulated networks although they are mostly obscured by the connecting lines.

The $\left(R_{2, \text { diff }}\right)^{2}$ and $R_{2}{ }^{\prime}$ values fitted from the log-ratios are plotted as a function of $\tau$ in Supplementary Fig. S4. The apparent $\left(R_{2, d i f f}\right)^{2}$ values obtained from fits of $\log \left(S_{\mathrm{SE}} / S_{\mathrm{ASE}}\right)$ vs. TE were in poor agreement with those from the fits of $\log \left(S_{\mathrm{SE}}\right) \mathrm{vs}$. TE; however, of consequence for calibration, the $R_{2}{ }^{\prime}$ values were within 5\% between the two fittings for the intermediate-to-large vessels. The apparent $\left(R_{2, d i f f}\right)^{2}$ and $R_{2}{ }^{\prime}$ values at $\tau=30 \mathrm{~ms}$ were then compared at more CBVs and $\Delta \chi$ values and plotted in Fig. S5. It was found that both $\left(R_{2, d i f f}\right)^{2}$ and $R_{2}{ }^{\prime}$ followed functions of the form

$$
\begin{gathered}
\left(R_{2, \text { diff }}\right)^{2}=a \operatorname{CBV}(\Delta \chi)^{b} \\
\text { and } \\
R_{2}^{\prime}=c \operatorname{CBV}(\Delta \chi)^{\beta}
\end{gathered}
$$

where $a$ and $c$ are coefficients that depend on field strength, $\beta$ is the conventional exponent used in calibrated fMRI, and $b$ is the equivalent exponent for $\left(R_{2, \text { diff }}\right)^{2}$. Both $\left(R_{2, \text { diff }}\right)^{2}$ and $R_{2}{ }^{\prime}$ were linearly proportional to $\mathrm{CBV}$ and $b$ and $\beta$ were remarkably similar; both were approximately 2 when the radius was $1 \mu \mathrm{m}$ and decreased to $\sim 1.1-1.2$ for the $100-\mu \mathrm{m}$ simulations.

The log-ratios are shown for the Lauwers and Frechet vessel-size distributions in Fig. 9. These results are consistent with those of the individual radii above. The error bar sizes vary between the two distributions because there were far more vessels per network in the Lauwers simulations than the Frechet simulations. The vertical offset between the two curves reflects the 
intrinsic differences in $R_{2}{ }^{\prime}$ between the two vessel-size distributions; the Frechet distribution has larger ratios because its vessel sizes are larger overall. The slope of the Lauwers curve at the smallest $\tau$ is initially steeper than that of the Frechet curve but it plateaus at the later echo times due to its smaller vessel size composition.

(a)

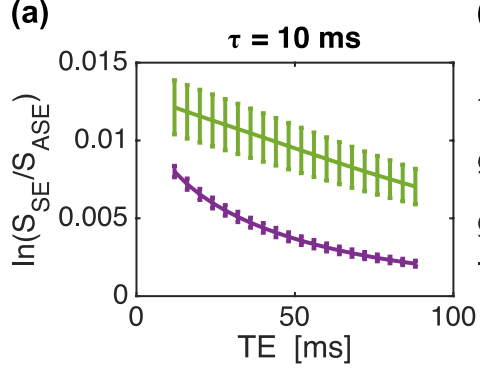

(b)

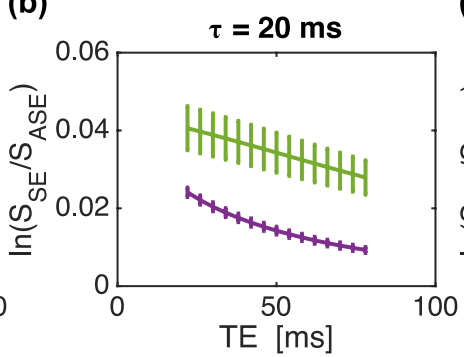

(c)

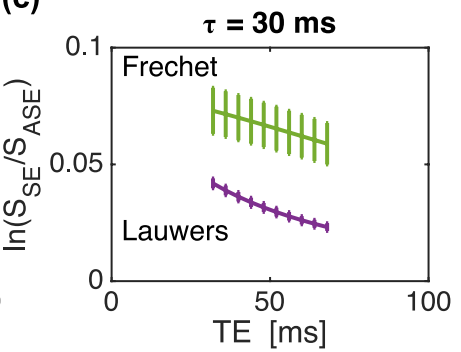

Fig. 9: The log-ratio of the SE signal $(\tau=0 \mathrm{~ms})$ over the ASE signal for three different ASE offsets, as a function of TE and for the Lauwers and Frechet vessel-size distributions. The distributions are labelled in (c). Error bars represent the mean \pm the standard deviation of the log-ratio across all simulated networks.

Fig. 10 shows the results of calculating $M$ using the ratio $\ln \left(S_{\mathrm{SE}} / S_{\mathrm{ASE}}\right)$ at a TE of $40 \mathrm{~ms}$ with $\tau=30 \mathrm{~ms}$ (denoted $M_{\mathrm{ASE}}$ ). When the ratio from TE $=50 \mathrm{~ms}$ was incorporated to calculate $R_{2}^{\prime}$ and $\left(R_{2, d i f f}\right)^{2}$ using the q-ASE model, almost all radii's $M$ values were substantially increased (denoted $M_{\mathrm{q}-\mathrm{ASE}}$ ). These ASE-based $M$ values were compared against the "ideal" $M$ value $\left(M_{\text {ideal }}\right)$, calculated as the maximum possible GE BOLD percent signal change at TE $=30 \mathrm{~ms}$. $M_{\mathrm{ASE}}$ only agreed to within $5 \%$ of $M_{\text {ideal }}$ for radii $>40 \mu \mathrm{m}$, whereas for $M_{\mathrm{q}-\mathrm{ASE}}$, this level of agreement was attained for radii $>7 \mu \mathrm{m}$. The corresponding estimated $\left(R_{2, \text { diff }}\right)^{2}$ values are shown in Fig. 10b.

\subsubsection{Intrinsic $T_{2}$ Decay}

Similar results as the single-radii $M$ calculations above were observed in the Lauwers and Frechet radius distributions, plotted in Fig. 11. To be consistent with the in vivo acquisition, 
more echo times were incorporated into the q-ASE model. The $\left(R_{2, \text { diff }}\right)^{2}$ and $M$ estimates decreased when more echo times were incorporated with the Lauwers distribution but they were relatively unchanged in the Frechet distribution. The impact of adding intrinsic $T_{2}$ decay to the simulations is also shown in Fig. 11. $M$ from the (q-)ASE fits and $\left(R_{2, \text { diff }}\right)^{2}$ were effectively unchanged when $T_{2}$ decay was added. Conversely, $M_{\text {ideal }}$ increased significantly for both distributions. In additional testing not shown, it was found that the increase in $M_{\text {ideal }}$ was not the result of adding the intrinsic $T_{2}$ of tissue but was the result of the intravascular BOLD signal arising from the complete saturation of venous blood.

As shown in Supplementary Figs. S7 and S8, when random noise of varying levels was added to the simulations, the linear relationship of $\log (\mathrm{SE} / \mathrm{ASE})$ was quickly obscured. Despite this, the mean $\left(R_{2, d i f f}\right)^{2}$ and $M$ values were stable across different SNR levels but the variance of their estimates increased with noise level.

(a)

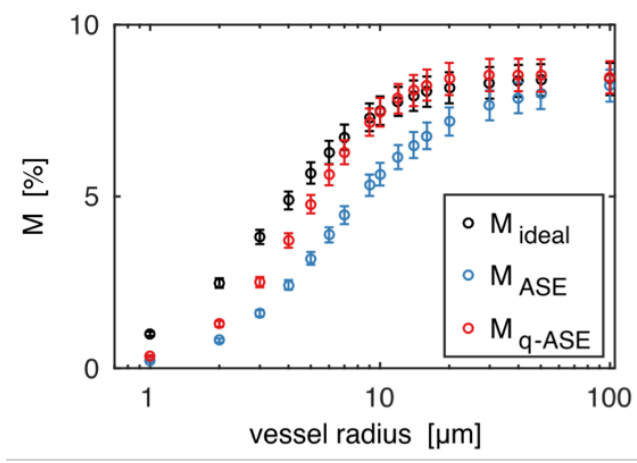

(b)

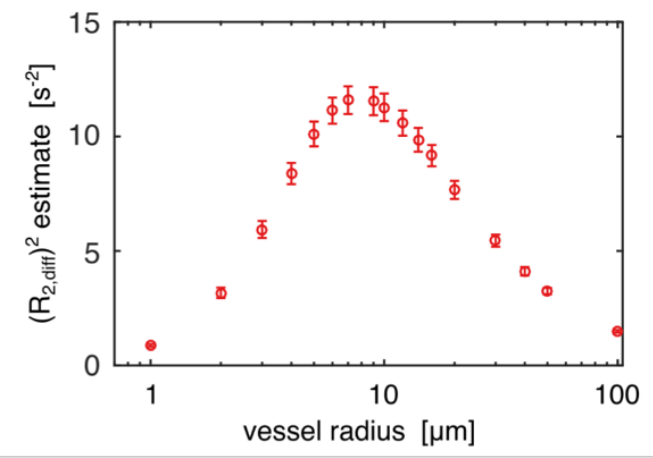

Fig. 10: (a) Radius-wise comparison of the ideal $M$ values $\left(M_{\text {ideal }}\right)$ calculated from the maximum GE BOLD signal at TE $=30 \mathrm{~ms}$ vs. $M$ when calculated using $\ln \left(S_{\mathrm{SE}} / S_{\mathrm{ASE}}\right)$ at a TE of $40 \mathrm{~ms}$ with $\tau$ $=30 \mathrm{~ms}\left(M_{\mathrm{ASE}}\right)$ and $M$ when calculated using the quadratic ASE signal model $\left(M_{\mathrm{q}-\mathrm{ASE}}\right)$ to fit for $R_{2}{ }^{\prime}$ and $\left(R_{2, \text { diff } f}\right)^{2}$ with SE and ASE signals at TE $=40$ and $50 \mathrm{~ms}$. (b) The estimated $\left(R_{2, \text { diff }}\right)^{2}$ values from the q-ASE model for each vessel radius. Error bars represent the mean \pm the standard deviation across all simulated networks. 

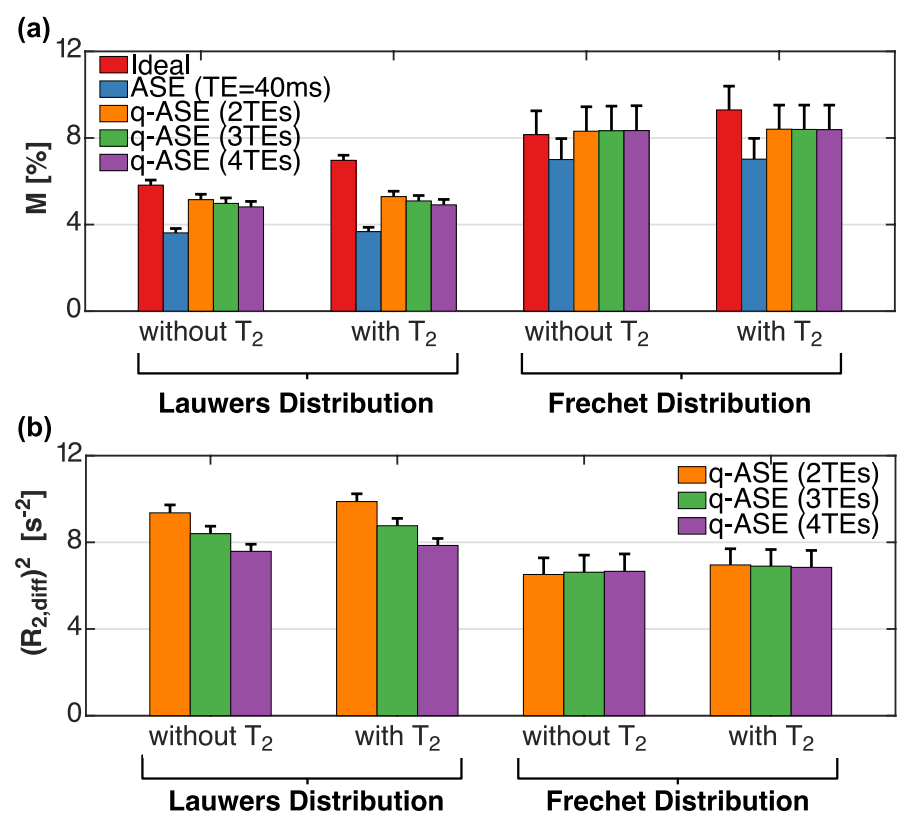

Fig. 11: Impact of intrinsic $T_{2}$ decay and number of echo times included in the q-ASE model on $M(\mathrm{a})$ and $\left(R_{2, \text { diff }}\right)^{2}$ (b) of the Lauwers and Frechet vessel size distributions. The ASE echo time was $40 \mathrm{~ms}$, and the q-ASE model was fit at echo times of 40, 50, 60, and $70 \mathrm{~ms}$ using the first $n=$ two to four echo times ( $n$ TEs).

\subsection{In Vivo Imaging}

The ratios of the SE and ASE signals, $\ln \left(S_{\mathrm{SE}} / S_{\mathrm{ASE}}\right)$, were calculated for all subjects from the image intensities averaged across the GM ROIs. In one of the subjects, these ratios displayed substantially more variability than in the other subjects resulting in this subject being excluded from any analyses. The ratios from the remaining subjects are shown in Fig. 12 along with their model fits to Eq. (7). Included in Fig. 12 are the average model fits derived from the mean of all subjects' fitted $R_{2}{ }^{\prime}$ and $\left(R_{2, d i f f}\right)^{2}$. Those fits are tabulated in Table 1.

The ASL data from another subject were excluded due to improper labelling plane positioning. The remaining 7 subjects' mean changes in $\mathrm{P}_{\mathrm{ET}} \mathrm{CO}_{2}$ and $\mathrm{P}_{\mathrm{ET}} \mathrm{O}_{2}$ during the hypercapnia challenge were $9 \pm 2 \mathrm{~mm} \mathrm{Hg}$ and $15 \pm 5 \mathrm{~mm} \mathrm{Hg}$, respectively. The gas delivery was not iso-oxic although this would only give an average change in arterial $\mathrm{SO}_{2}$ from approximately 
$97 \%$ at rest to $98 \%$ during hypercapnia (Severinghaus, 1979), resulting in a negligible contribution to the BOLD signal.

Each individual subjects' and the mean across subjects' calibration constants in the GM ROIs, as determined from hypercapnia $\left(M_{\mathrm{HC}}\right), \mathrm{ASE}$ at TE $=42 \mathrm{~ms}\left(M_{\mathrm{ASE}}\right), \mathrm{q}-\mathrm{ASE}$ fit with the first three TEs $\left(M_{\mathrm{q}-\mathrm{ASE}}(3 \mathrm{TEs})\right)$, and q-ASE fit with all four TEs $\left(M_{\mathrm{q}-\mathrm{ASE}}(4 \mathrm{TEs})\right)$ are displayed in Fig. 12.
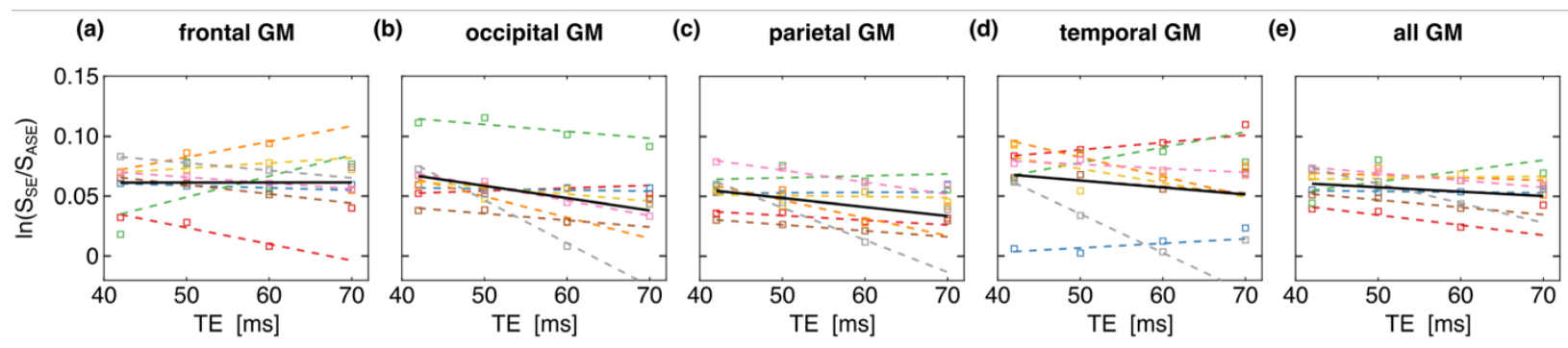

Fig. 12: The log-ratio of the in vivo SE signal over the ASE signal as a function of TE for the grey matter (GM) ROIs. Individual subjects' values are plotted with the coloured markers and their resulting fits to Eq. (7) with the first three TEs are plotted with the coloured dashed lines. The mean fits are represented by the solid black lines. All axes share the same set of axis labels as (a).

Table 1: The mean \pm SD in vivo $\left(R_{2, \text { diff }}\right)^{2}$ values across the grey matter (GM) ROIs of 8 subjects. The fits for $\left(R_{2, \text { diff }}\right)^{2}$ were performed using either the first three echoes or all four echoes.

\begin{tabular}{cccccc}
\hline $\begin{array}{c}\text { \# of Echoes } \\
\text { in q-ASE Fit }\end{array}$ & Frontal GM & Occipital GM & Parietal GM & Temporal & All Grey \\
& & & & GM & Matter \\
\hline 3 & $-0.1 \pm 18$ & $17 \pm 20$ & $12 \pm 16$ & $10 \pm 24$ & $6 \pm 12$ \\
4 & $-1 \pm 11$ & $9 \pm 10$ & $6 \pm 7$ & $3 \pm 15$ & $2 \pm 7$ \\
\hline
\end{tabular}


(a)

(b)
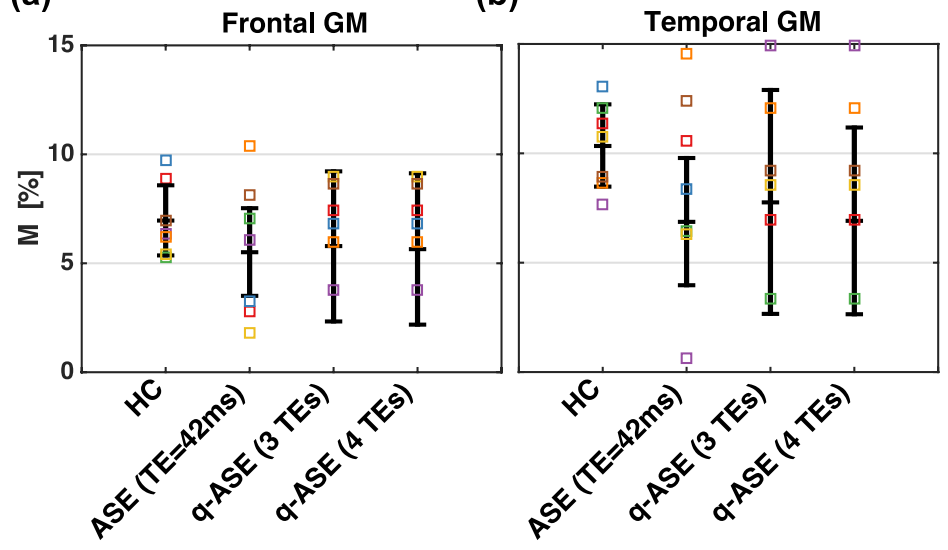

(c)

(d)

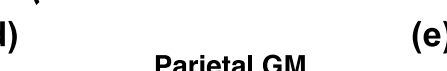

(e)
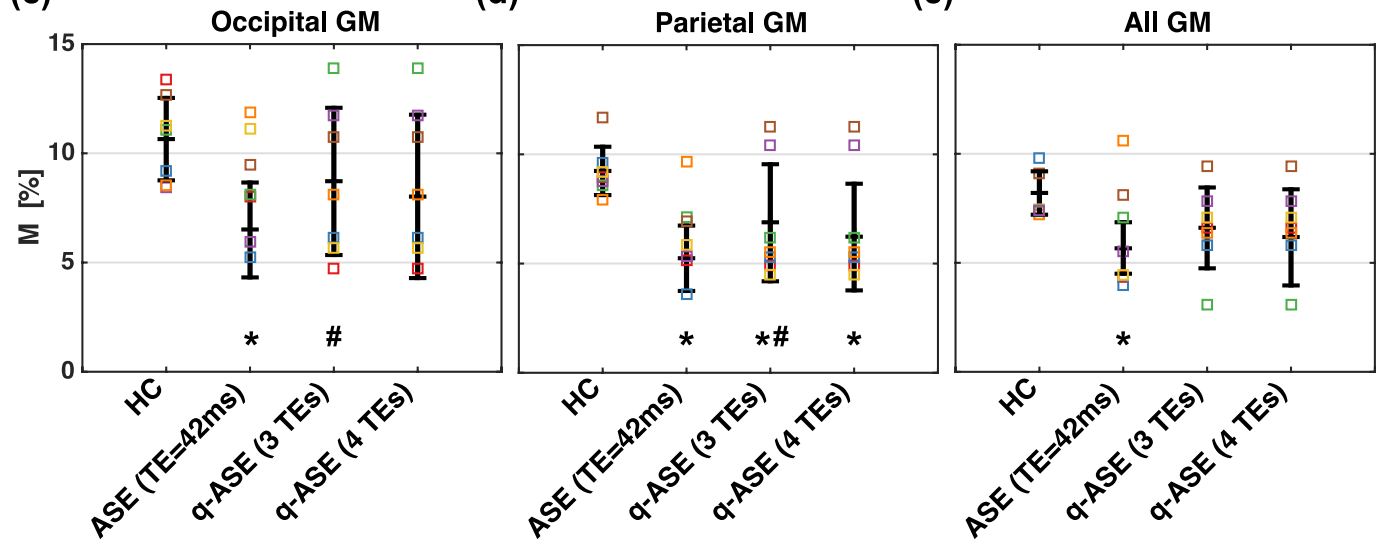

Fig. 13: Comparison of $M$ in the grey matter ROIs across seven subjects. $M$ was measured using hypercapnia (HC), from the first ASE echo time (ASE (TE=42 ms)), with the q-ASE model fit to the first three TEs (q-ASE (3 TEs)), or all four TEs (q-ASE (4 TEs)). Each individual subjects' values are represented by the square symbols and a separate colour and the mean \pm SD across subjects is represented by the solid black line. * denotes the ASE or q-ASE $M$ values were significantly different from the HC values $(P<0.05)$. \# denotes the q-ASE $M$ values were significantly greater than the ASE $(\mathrm{TE}=42 \mathrm{~ms}) M$ values $(P<0.05)$.

\section{Discussion}

Gas-free fMRI calibration holds great appeal for improving access to calibrated fMRI methods; however, technical challenges still impede its adoption. In this study, we have examined SE attenuation resulting from diffusion through the microvasculature with the principal aim of being able to compensate for this attenuation in calibration. Despite SE attenuation being a well-known 
phenomenon, it has generally been disregarded in earlier studies using gas-free calibration (Fujita et al., 2006; Kida et al., 2000; Shu et al., 2015) or acknowledged as a limitation of current implementations (Blockley et al., 2015). Here, we have shown that the attenuation substantially reduces the calibration constant when estimated with ASE imaging, in line with previous analytical simulations and in vivo measurements (Blockley et al., 2015), but that the underestimation can be compensated by acquiring additional ASE images.

\subsection{Microvascular Simulations}

Using simulations from networks of vessels with identical radii and from two different distributions of radii, the general nature of the SE attenuation became much more apparent. By empirically describing the attenuation by a quadratic-exponential decay early on and a linearexponential decay later, it was possible to identify several features of it: i) the overall magnitude of the attenuation is largest for intermediate vessel sizes; ii) the time to transition from quadratic to linear decay is proportional to vessel size for the small-to-intermediate radii and peaks or plateaus for larger radii; and iii) the attenuation at later ASE offsets $(\tau \neq 0)$ can approximately be described by the SE ( $\tau=0$ ) attenuation but translated in time by $\tau$ and with an additional offset given by the intrinsic $R_{2}{ }^{\prime}$ weighting. The first feature is already well known from SE BOLD vessel-size sensitivity studies (Boxerman et al., 1995; Goense and Logothetis, 2006; Zhao et al., 2004). The second and third features are novel findings of this study and led us to propose the quadratic ASE (q-ASE) biophysical signal model, described by Eqs. (5)-(7), with the addition of the diffusion-induced attenuation constant, $\left(R_{2, \text { diff }}\right)^{2}$. This model correctly predicts that $\ln \left(S_{\mathrm{SE}} / S_{\mathrm{ASE}}\right)$ underestimates $M$, and, crucially, it allows for the underestimation to be quantified by measuring the SE and ASE signals at two or more TEs. A model where the decay exponent was not exactly 2 but was empirically determined was considered; however, it was decided to keep the exponent at 2 based on the simplicity with which it allows the fitting of $\ln \left(S_{\mathrm{SE}} / S_{\mathrm{ASE}}\right)$ vs. TE and based on the success with which it corrected the $M$ values in simulations. In contrast to the simulations here, previous analytical calculations using a detailed BOLD signal model accounted for SE attenuation by modelling it as a linear exponential decay (Blockley et al., 2012; Blockley et al., 2015). Those calculations also predicted an underestimation of $M$, however, as 
discussed in the Theory, the linear decay does not provide a means by which it can be easily corrected.

A consequence of the transition from quadratic to linear decay is that the window of time to characterize the SE attenuation is limited: once the signal transitions to linear-exponential decay, the attenuation becomes indistinguishable from intrinsic $R_{2}$ and gives a decreased apparent $R_{2}^{\prime}$ (and, therefore, decreased $M$ ). This is evident when comparing the estimated $\left(R_{2, \text { diff }}\right)^{2}$ values from the simulations in Fig. 10b with the true $\left(R_{2, \text { diff }}\right)^{2}$ values in Fig. 7 . The true $\left(R_{2, \text { diff }}\right)^{2}$ values are largest for the smaller radii and decrease monotonically with increasing radius, however, the estimated $\left(R_{2, \text { diff }}\right)^{2}$ values peak for the intermediate vessel sizes. Because of this, the q-ASE model is still unable to effectively compensate for the underestimation in $M$ for the smallest vessel sizes, whereas it performs remarkably well for intermediate to large radii. Physiologically, this would correspond to the correction working for most post-capillary vessels if one takes the upper cut-off radius for capillaries to be 4-5 $\mu \mathrm{m}$ (Gagnon et al., 2015; Lauwers et al., 2008; Pawlik et al., 1981; Sakadzic et al., 2014; Stefanovic et al., 2008). $\left(R_{2, d i f f}\right)^{2}$ was further characterized by its dependence on CBV and $\Delta \chi$ in the Supplementary Materials. There, it was shown that it behaved much like how $R_{2}{ }^{\prime}$ is known to; it was linearly proportional to CBV and non-linearly proportional to $\Delta \chi$ with an exponent that was very close to the conventional $\beta$ value.

The q-ASE modelling on the simulations from the vessel-size distributions was relatively unchanged when $T_{2}$ decay was added. This is reassuring as this was a principal motivating factor for using the ASE imaging method.

\subsection{In Vivo Imaging}

Encouraged by the simulation results, we sought to determine the feasibility of measuring $\left(R_{2, \text { diff }}\right)^{2}$ in vivo and to compensate for the SE attenuation during calibration. We measured SE and ASE images ( $\tau=30 \mathrm{~ms}$ ) at TEs of 42, 50, 60, and $70 \mathrm{~ms}$ with the CSF signal nulled to avoid its contamination of $R_{2}{ }^{\prime}$. Anticipating that the ratio of the SE over the ASE signal could plateau at later echo times, we fit for $\left(R_{2, d i f f}\right)^{2}$ and $R_{2}{ }^{\prime}$ using either the first three TEs or all four. 
Independent of the number of TEs, there was considerable variability in the fits across subjects that resulted in positive and negative values for $\left(R_{2, \text { diff }}\right)^{2}$; however, for all but the frontal GM ROI, the mean fits resulted in positive $\left(R_{2, \text { diff }}\right)^{2}$ (Table 1). The mean measured values were in line with those predicted by the simulations and the associated variability was consistent with the simulations when noise was added to them (see Supplementary Figs. S7 and S8).

The mean $\left(R_{2, \text { diff }}\right)^{2}$ values were larger for the three-TE fits vs. the four-TE fits, perhaps implying that $\ln \left(S_{\mathrm{SE}} / S_{\mathrm{ASE}}\right)$ does plateau beyond $\mathrm{TE}=60 \mathrm{~ms}$. The resulting increases in $M$ were significant in the occipital and parietal ROIs for the three-TE fit but not the four-TE fit, further suggesting that the data at the later echoes should not be used (Fig. 13). Based on the simulations from the two different vessel-size distributions, the Lauwers distribution showed a similar decrease in $M$ when later echoes were included in the fits, as shown in Fig. 11. This may suggest that that distribution is more representative of the venous/capillary vessel sizes in grey matter. Relative to hypercapnia, the ASE-based $M$ estimates all underestimated $M_{\mathrm{HC}}$, although the underestimation was not statistically significant for all comparisons. When compared against simulations with or without intrinsic $T_{2}$ decay, the ideal $M$ values increased when $T_{2}$ decay was included such that the gaps between the ideal $M$ and the (q-)ASE $M$ calculations all widened. The increase in the ideal $M$ values was due almost entirely to the intravascular BOLD signal. This is consistent with current knowledge of the BOLD signal where it is known that IV signal has a significant contribution to the BOLD effect at field strengths of $1.5 \mathrm{~T}$ and $3 \mathrm{~T}$ because of hydrogen nuclei's proximity to hemoglobin (Donahue et al., 2011).

Multiple reasons factored into the decision to use a single ASE offset in vivo. Practically, ASE and SE images were acquired at four different TE values, resulting in the acquisition time being just over 18 minutes. Adding one more tau value would have increased the acquisition time to over $27 \mathrm{~min}$ and was considered impractical. Theoretically, it has recently been shown by Stone and Blockley (2016) that using larger tau values - comparable to the lower of $T_{2}$ or $T_{2}^{\prime}-$ will improve the accuracy of $R_{2}{ }^{\prime}$ estimates. This was derived using the conventional ASE model, ignoring the effect of diffusion. From our simulations, however, the q-ASE model held at lower echo times and was likely to lose its applicability beyond TE $\sim 70 \mathrm{~ms}$. Given this, a trade-off between obtaining a biased estimate of $R_{2}{ }^{\prime}$ with increased precision vs. an accurate estimate of 
$R_{2}{ }^{\prime}$ and $\left(R_{2, \text { diff }}\right)^{2}$ with lower precision existed. A value of $\tau=30 \mathrm{~ms}$ was settled on, such that the signal difference between $\tau=0$ and $30 \mathrm{~ms}$ could be sufficiently measured and a range of shorter TEs could be used.

Note that although $M_{\mathrm{HC}}$ was used for comparison, this does not imply that it is the standard for calibration. As discussed in the Introduction, hypercapnic calibration suffers from its own setbacks and technical challenges, including what exact values to use for $\alpha$ and $\beta$ (Gagnon et al., 2016; Griffeth and Buxton, 2011). This means that the true magnitude of the underestimation of $M$ in the ASE methods could be less (or more) than implied by the comparison against $M_{\mathrm{HC}}$. Furthermore, if $\mathrm{CMRO}_{2}$ were to decrease during the hypercapnic challenge, then the true $M_{\mathrm{HC}}$ would be less than the estimated value and could account for a significant fraction of the discrepancy (Driver et al., 2017; Xu et al., 2011). An alternative measurement of $R_{2}{ }^{\prime}$ may be a more appropriate comparison. This could consist of separate multi-echo GE and SE acquisitions, using a relatively short SE spacing to reduce the impact of diffusion-induced attenuation. In this case, multi-exponential decay would complicate the comparison but a correction could be incorporated (Fujita et al., 2006).

When compared against the study by Blockley et al. (2015), the measured $M_{\text {ASE }}$ values relative to $M_{\mathrm{HC}}$ values are consistent. In that study, SE and ASE images were acquired at a single TE of $40 \mathrm{~ms}$ with a spiral readout with a marginally higher in-plane resolution, the same slice thickness, no CSF suppression, and similar post-processing steps. The mean ratio of $M_{\mathrm{ASE}} / M_{\mathrm{HC}}$ across all GM was $\approx 0.9$ in that study and $\approx 0.7$ here for the $42-\mathrm{ms} \mathrm{TE}$ data. The reduced ratio was expected in our study because CSF suppression has been shown to reduce $R_{2}{ }^{\prime}$ estimates in GM by $20-30 \%$ (Simon et al., 2016; Stone and Blockley, 2016). This further underscores the importance that SE attenuation likely plays in the resulting underestimation of $R_{2}{ }^{\prime}$ and $M$.

\subsection{Biophysical Signal Modelling}

The q-ASE signal model presented here is complementary to biophysical signal models that propose a Gaussian signal characteristic about the spin echo (He and Yablonskiy, 2007; Mulkern et al., 2014; Yablonskiy and Haacke, 1994). These signal models typically assume perfect SE 
refocusing and describe a Gaussian-like decay around the spin echo with respect to TE or $\tau$, whereas the q-ASE model describes a Gaussian-like decay of the spin echo itself as a function of TE. These Gaussian SE signal characteristics are still present in our simulations (see the individual time series in Fig. 2) since the simulations used no assumptions about the underlying frequency distribution shape (e.g. Lorentzian or Gaussian) other than that the individual fields produced by the vessels are modelled as dipoles from infinite cylinders. The quantitative BOLD model is one such model of Gaussian signal decay around the $\mathrm{SE}$, and it is used to relate $R_{2}{ }^{\prime}$ to deoxygenated-CBV and $\mathrm{SO}_{2}$ (He and Yablonskiy, 2007). Since this model ignores the effects of diffusion, it may benefit from the q-ASE model by correcting its estimate of $R_{2}^{\prime}$.

\subsection{Limitations and Future Work}

An important limitation of this study is that it ignored the contributions of non-heme sources of susceptibility to $R_{2}{ }^{\prime}$ and it did not show any causal relationship between the measured $\left(R_{2, \text { diff }}\right)^{2}$ and $R_{2}{ }^{\prime}$ parameters and blood oxygenation. The concentration of non-heme iron is known to increase in many brain regions during healthy aging, including in deep GM primarily but also in cortical GM (Hallgren and Sourander, 1958). $R_{2}, R_{2}{ }^{*}, R_{2}{ }^{\prime}$ and susceptibility have all been shown to correlate with post-mortem regional iron concentrations (Li et al., 2014; Ordidge et al., 1994; Sedlacik et al., 2014; Stuber et al., 2014). The $R_{2}{ }^{\prime}$ values in cortical GM from Sedlacik et al. (2014) were not in agreement with the linear regression performed on the subcortical GM $R_{2}{ }^{\prime}$ values vs. the literature iron concentrations of Hallgren and Sourander (1958). This may suggest that the $R_{2}^{\prime}$ of cortical GM is less affected by iron deposition over the course of healthy aging. In post hoc testing, none of the percent differences between $M_{\mathrm{HC}}$ and $M_{\mathrm{q}-\mathrm{ASE}}(3 \mathrm{TEs})$ were statistically significant when regressed against subject age (from 19 to 39 years). This could be because both $M \mathrm{~s}$ were most strongly dependent on vascular physiology than on non-heme sources but the small cohort size might also limit the measurability of true differences with age. While beyond the scope of this study, these factors could be teased apart by repeating the ASE imaging experiments during a hypercapnia or hyperoxia challenge. In doing this, the heme contributions to $R_{2}{ }^{\prime}$ and $\left(R_{2, \text { diff }}\right)^{2}$ would decrease (for increasing $\mathrm{SO}_{2}$ ) and the non-heme contributions would be unchanged. Given the large variability of $\left(R_{2, \text { diff } f}\right)^{2}$ measured here, either 
the imaging technique would require further refinement to be more sensitive to the small changes

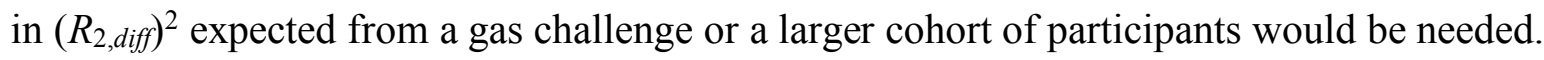

An additional limitation of estimating $M$ from a measure of $R_{2}{ }^{\prime}$ at rest is that it does not capture IV BOLD signal, as discussed above. It may be possible to circumvent the IV BOLD issue by applying IV crushers during the subsequent functional experiments (An and Lin, 2003; Hare and Bulte, 2016), although this will decrease the detection sensitivity.

Despite considerable efforts to reduce $R_{2}{ }^{\prime}$ contributions from macroscopic field inhomogeneities in vivo, the results in the frontal and temporal ROIs were still inconsistent with the results in the occipital and parietal ROIs. The $M$ values in the frontal and temporal ROIs were in the range of the other ROIs', suggesting that the bulk effects of the field inhomogeneities were largely avoided; however, the increase in $M$ from the q-ASE model failed to reach statistical significance in the frontal and temporal ROIs, suggesting that there was increased signal variability in these regions that could arise from residual field effects. In future studies, further increasing the spatial resolution and/or performing z-shimming could help alleviate these issues but at the expense of increased readout and/or scan time (Ordidge et al., 1994).

Efforts to minimize the impact of macroscopic inhomogeneities on $R_{2}^{\prime}$ could possibly explain some of the underestimation of $M$ relative to hypercapnia since signal loss from longranging field gradients originating from large veins will be reduced if the voxel size is reduced (Turner, 2002). These effects will also be dependent on the orientation of the cortical surface relative $B_{0}$ (Gagnon et al., 2015). Our simulations did not account for any macroscopic or mesoscopic non-random vessel orientation effects, although it has been shown that the GE BOLD effect has a strong dependence on cortical orientation relative to $B_{0}$ because of longranging field gradients from pial veins (Gagnon et al., 2015). The orientation effects were significantly less for SE BOLD relative to GE BOLD (Gagnon et al., 2015), therefore, we do not expect the quadratic ASE attenuation described here to be substantially impacted by non-random vessel orientation. Consequently, while baseline $R_{2}{ }^{\prime}$ may be influenced by cortical orientation, the quantification of the SE attenuation should not be significantly impacted. 
Ideally, one would like to apply the q-ASE correction using an individual's measured $\left(R_{2, \text { diff }}\right)^{2}$ parameter. Unfortunately, this measurement added considerable time to the scan and it was quite variable on the individual subject-level - requiring the data from one subject to be excluded from analysis. Although $\left(R_{2, d i f f}\right)^{2}$ depends on $\mathrm{CBV}$ and $\mathrm{SO}_{2}$, it may be more beneficial to acquire SE and ASE images at a single TE and to apply the q-ASE correction using an assumed $\left(R_{2, \text { diff }}\right)^{2}$ value or by relating it to the apparent $R_{2}{ }^{\prime}$ since they were found to have similar dependences on $\mathrm{CBV}$ and $\mathrm{SO}_{2}$. This would be akin to the calibrated fMRI parameter $\beta$, which could be measured in vivo (Croal et al., 2017; Shu et al., 2016) but is generally assumed a constant across the brain. Considering the $\left(R_{2, \text { diff }}\right)^{2}$ values from the simulations and the in vivo measurements, a value around $10-15 \mathrm{~s}^{-2}$ may be appropriate.

Here, we have presented a comparison of different methods for estimating the calibration constant, $M$, with the viewpoint that this is a tissue parameter - effectively, a scaling of $R_{2}{ }^{\prime}$ and dependent on $\mathrm{CBV}, \mathrm{SO}_{2}$, vessel size, field strength, etc. However, the goal in a calibrated fMRI experiment is to measure changes in $\mathrm{CMRO}_{2}$ using the measured $M$ parameter. Recent simulation studies of the calibration process suggest that the final application of the calibration model depends on how calibration was performed and requires optimized values of the parameters $\alpha$ and $\beta$ to obtain the most accurate measure of $\mathrm{CMRO}_{2}$ changes (Gagnon et al., 2016; Griffeth and Buxton, 2011). This necessitates dropping the original biophysical associations of $M, \alpha$, and $\beta$ and rather using them as empirical model-dependent parameters. Considering this, the $\alpha$ and $\beta$ values used for hypercapnic calibration may not be optimal for calibrated fMRI using a gas-free estimate of $M$ and they should be evaluated and optimized in future work.

The concept of the model dependence of $M$ was extended by Simon et al. (2016), where $M$ was never explicitly calculated but an estimate of resting $R_{2}{ }^{\prime}$ was used in a Bayesian model to calculate changes in $\mathrm{CMRO}_{2}$ in subsequent functional experiments. $R_{2}{ }^{\prime}$-based calibration in their study was found to be sensitive to several parameters, including imperfect spin echo refocusing in the capillary bed. Their input signal model assumed perfect SE refocusing beyond the capillary bed but our results show that the SE attenuation in intermediate sized venules is still substantial and should not be ignored. With the characterization of the vessel-size and $\mathrm{SO}_{2}$ 
dependence of $\left(R_{2, \text { diff }}\right)^{2}$ performed here, incorporation of a signal model with $\left(R_{2, \text { diff }}\right)^{2}$ could potentially reduce the Bayesian model's sensitivity to imperfect SE refocusing, not only for capillaries but for larger post-capillary venules too. Conversely, application of the Bayesian model to $\left(R_{2, \text { diff }}\right)^{2}$ measurements could help reduce the error propagation from the noisy measurement encountered here. It should be noted that that study measured $R_{2}{ }^{\prime}$ with two gradient echo sampling of the spin echo (GESSE) sequences - one with the SE occurring at $48 \mathrm{~ms}$ and the other at $98 \mathrm{~ms}$. Two SE times were used to account for multi-exponential decay. We have shown here that even SE times of $40 \mathrm{~ms}$ will suffer from SE attenuation, therefore these GESSE sequences would underestimate $R_{2}{ }^{\prime}$; however, as discussed above, incorporating a model of SE attenuation may help alleviate this. The early SE time could be used to correct for the SE attenuation, and the later SE time could still be used in the multi-exponential decay correction.

\section{Conclusions}

In this study, we sought to characterize the attenuation of SE and ASE signals arising from diffusion of water surrounding the microvasculature. Using simulations, we have shown how this attenuation varies for different vessel sizes, blood volumes, and susceptibility offsets, and how it impacts gas-free calibrated fMRI based on ASE imaging. We have proposed that the initial attenuation be described as a quadratic-exponential decay term, $\left(R_{2, \text { diff }}\right)^{2}$, such that it can be measured and compensated for by acquiring SE and ASE signals at multiple echo times. This strategy successfully corrected the underestimation of the fMRI calibration constant in the simulations for intermediate vessel radii and above. By replicating these experiments in vivo in healthy subjects, we showed that the $M$ values obtained from the ratio of the SE and ASE images at a single TE could be significantly increased by incorporating the ratios from later TEs; however, they still tended to underestimate the $M$ values obtained from the more common hypercapnic calibration. Future studies of gas-free calibration and $R_{2}{ }^{\prime}$ imaging will benefit from incorporating an estimation of $\left(R_{2, d i f f}\right)^{2}$ in their fitting or using an assumed value to compensate for the underestimation of $R_{2}{ }^{\prime}$. 


\section{Acknowledgements}

The authors would like to thank Drs. Felipe Tancredi and Richard Hoge for sharing their gas delivery system software and hardware schematics. The simulations in this study were enabled in part by computing support provided by WestGrid (www.westgrid.ca) and Compute Canada Calcul Canada (www.computecanada.ca). This work was supported by the Canadian Institutes of Health Research [FDN 143290] and the Campus Alberta Innovates Program. 


\section{$\underline{\text { References }}$}

An, H., Lin, W., 2003. Impact of intravascular signal on quantitative measures of cerebral oxygen extraction and blood volume under normo- and hypercapnic conditions using an asymmetric spin echo approach. Magn Reson Med 50, 708-716.

Ashburner, J., Friston, K.J., 2005. Unified segmentation. Neuroimage 26, 839-851.

Bandettini, P.A., Wong, E.C., 1995. Effects of Biophysical and Physiological-Parameters on Brain Activation-Induced R(2)Asterisk and R(2) Changes - Simulations Using a Deterministic Diffusion-Model. International Journal of Imaging Systems and Technology 6, 133-152.

Berman, A.J., Ma, Y., Hoge, R.D., Pike, G.B., 2016. The effect of dissolved oxygen on the susceptibility of blood. Magn Reson Med 75, 363-371.

Berman, A.J., Pike, G.B., 2016. Breaking beta: Understanding the beta-value in calibrated fMRI. 24th International Society of Magnetic Resonance in Medicine Annual Meeting, Singapore, p. 506.

Berman, A.J.L., Pike, G.B., 2017. Transverse signal decay under the weak field approximation: Theory and validation. Magn Reson Med.

Blockley, N.P., Griffeth, V.E., Buxton, R.B., 2012. A general analysis of calibrated BOLD methodology for measuring $\mathrm{CMRO} 2$ responses: comparison of a new approach with existing methods. Neuroimage 60, 279-289.

Blockley, N.P., Griffeth, V.E., Simon, A.B., Dubowitz, D.J., Buxton, R.B., 2015. Calibrating the BOLD response without administering gases: comparison of hypercapnia calibration with calibration using an asymmetric spin echo. Neuroimage 104, 423-429.

Blockley, N.P., Stone, A.J., 2016. Improving the specificity of R2' to the deoxyhaemoglobin content of brain tissue: Prospective correction of macroscopic magnetic field gradients. Neuroimage 135, 253-260.

Boxerman, J.L., Hamberg, L.M., Rosen, B.R., Weisskoff, R.M., 1995. MR Contrast Due to Intravascular Magnetic-Susceptibility Perturbations. Magn Reson Med 34, 555-566.

Bulte, D.P., Chiarelli, P.A., Wise, R.G., Jezzard, P., 2007. Cerebral perfusion response to hyperoxia. J Cereb Blood Flow Metab 27, 69-75.

Bulte, D.P., Drescher, K., Jezzard, P., 2009. Comparison of hypercapnia-based calibration techniques for measurement of cerebral oxygen metabolism with MRI. Magn Reson Med 61, 391-398.

Bulte, D.P., Kelly, M., Germuska, M., Xie, J., Chappell, M.A., Okell, T.W., Bright, M.G., Jezzard, P., 2012. Quantitative measurement of cerebral physiology using respiratory-calibrated MRI. Neuroimage 60, 582-591. 
Chen, J.J., Pike, G.B., 2009. Human whole blood T2 relaxometry at 3 Tesla. Magn Reson Med $61,249-254$.

Chen, J.J., Pike, G.B., 2010a. Global cerebral oxidative metabolism during hypercapnia and hypocapnia in humans: implications for BOLD fMRI. J Cereb Blood Flow Metab 30, 1094-1099.

Chen, J.J., Pike, G.B., 2010b. MRI measurement of the BOLD-specific flow-volume relationship during hypercapnia and hypocapnia in humans. Neuroimage 53, 383-391.

Chiarelli, P.A., Bulte, D.P., Wise, R., Gallichan, D., Jezzard, P., 2007. A calibration method for quantitative BOLD fMRI based on hyperoxia. Neuroimage 37, 808-820.

Croal, P.L., Driver, I.D., Francis, S.T., Gowland, P.A., 2017. Field strength dependence of grey matter R2 $\left.{ }^{*}\right)$ on venous oxygenation. Neuroimage 146, 327-332.

Croal, P.L., Hall, E.L., Driver, I.D., Brookes, M.J., Gowland, P.A., Francis, S.T., 2015. The effect of isocapnic hyperoxia on neurophysiology as measured with MRI and MEG. Neuroimage $105,323-331$.

Davis, T.L., Kwong, K.K., Weisskoff, R.M., Rosen, B.R., 1998. Calibrated functional MRI: mapping the dynamics of oxidative metabolism. Proc Natl Acad Sci USA 95, 1834-1839.

Deichmann, R., Josephs, O., Hutton, C., Corfield, D.R., Turner, R., 2002. Compensation of susceptibility-induced BOLD sensitivity losses in echo-planar fMRI imaging. Neuroimage 15, 120-135.

Donahue, M.J., Hoogduin, H., van Zijl, P.C., Jezzard, P., Luijten, P.R., Hendrikse, J., 2011. Blood oxygenation level-dependent (BOLD) total and extravascular signal changes and DeltaR2* in human visual cortex at 1.5, 3.0 and 7.0 T. NMR Biomed 24, 25-34.

Driver, I.D., Wise, R.G., Murphy, K., 2017. Graded Hypercapnia-Calibrated BOLD: Beyond the Iso-metabolic Hypercapnic Assumption. Front Neurosci 11, 276.

Fujita, N., Matsumoto, K., Tanaka, H., Watanabe, Y., Murase, K., 2006. Quantitative study of changes in oxidative metabolism during visual stimulation using absolute relaxation rates. NMR Biomed 19, 60-68.

Fujita, N., Shinohara, M., Tanaka, H., Yutani, K., Nakamura, H., Murase, K., 2003. Quantitative mapping of cerebral deoxyhemoglobin content using MR imaging. Neuroimage 20, 2071-2083.

Gagnon, L., Sakadzic, S., Lesage, F., Musacchia, J.J., Lefebvre, J., Fang, Q., Yucel, M.A., Evans, K.C., Mandeville, E.T., Cohen-Adad, J., Polimeni, J.R., Yaseen, M.A., Lo, E.H., Greve, D.N., Buxton, R.B., Dale, A.M., Devor, A., Boas, D.A., 2015. Quantifying the Microvascular Origin of BOLD-fMRI from First Principles with Two-Photon Microscopy and an OxygenSensitive Nanoprobe. J Neurosci 35, 3663-3675. 
Gagnon, L., Sakadzic, S., Lesage, F., Pouliot, P., Dale, A.M., Devor, A., Buxton, R.B., Boas, D.A., 2016. Validation and optimization of hypercapnic-calibrated fMRI from oxygen-sensitive two-photon microscopy. Philos Trans R Soc Lond B Biol Sci 371.

Gauthier, C.J., Hoge, R.D., 2012. Magnetic resonance imaging of resting OEF and CMRO(2) using a generalized calibration model for hypercapnia and hyperoxia. Neuroimage 60, 12121225 .

Gauthier, C.J., Madjar, C., Tancredi, F.B., Stefanovic, B., Hoge, R.D., 2011. Elimination of visually evoked BOLD responses during carbogen inhalation: implications for calibrated MRI. Neuroimage 54, 1001-1011.

Germuska, M.A., Meakin, J.A., Bulte, D.P., 2013. The influence of noise on BOLD-mediated vessel size imaging analysis methods. J Cereb Blood Flow Metab 33, 1857-1863.

Goense, J.B., Logothetis, N.K., 2006. Laminar specificity in monkey V1 using high-resolution SE-fMRI. Magn Reson Imaging 24, 381-392.

Greve, D.N., Fischl, B., 2009. Accurate and robust brain image alignment using boundary-based registration. Neuroimage 48, 63-72.

Griffeth, V.E., Buxton, R.B., 2011. A theoretical framework for estimating cerebral oxygen metabolism changes using the calibrated-BOLD method: modeling the effects of blood volume distribution, hematocrit, oxygen extraction fraction, and tissue signal properties on the BOLD signal. Neuroimage 58, 198-212.

Grubb, R.L., Jr., Raichle, M.E., Eichling, J.O., Ter-Pogossian, M.M., 1974. The effects of changes in $\mathrm{PaCO} 2$ on cerebral blood volume, blood flow, and vascular mean transit time. Stroke 5, 630-639.

Gudbjartsson, H., Patz, S., 1995. The Rician distribution of noisy MRI data. Magn Reson Med 34, 910-914.

Hall, E.L., Driver, I.D., Croal, P.L., Francis, S.T., Gowland, P.A., Morris, P.G., Brookes, M.J., 2011. The effect of hypercapnia on resting and stimulus induced MEG signals. Neuroimage 58, 1034-1043.

Hallgren, B., Sourander, P., 1958. The effect of age on the non-haemin iron in the human brain. J Neurochem 3, 41-51.

Hare, H.V., Bulte, D.P., 2016. Investigating the dependence of the calibration parameter M on echo time. Magn Reson Med 75, 556-561.

He, X., Yablonskiy, D.A., 2007. Quantitative BOLD: mapping of human cerebral deoxygenated blood volume and oxygen extraction fraction: default state. Magn Reson Med 57, 115-126. 
Hoge, R.D., Atkinson, J., Gill, B., Crelier, G.R., Marrett, S., Pike, G.B., 1999. Investigation of BOLD signal dependence on cerebral blood flow and oxygen consumption: the deoxyhemoglobin dilution model. Magn Reson Med 42, 849-863.

Jenkinson, M., Beckmann, C.F., Behrens, T.E., Woolrich, M.W., Smith, S.M., 2012. Fsl. Neuroimage 62, 782-790.

Jenkinson, M., Smith, S., 2001. A global optimisation method for robust affine registration of brain images. Med Image Anal 5, 143-156.

Jensen, J.H., Chandra, R., 2000. NMR relaxation in tissues with weak magnetic inhomogeneities. Magn Reson Med 44, 144-156.

Jezzard, P., Balaban, R.S., 1995. Correction for geometric distortion in echo planar images from B0 field variations. Magnetic Resonance in Medicine 34, 65-73.

Kida, I., Kennan, R.P., Rothman, D.L., Behar, K.L., Hyder, F., 2000. High-resolution CMR(O2) mapping in rat cortex: a multiparametric approach to calibration of BOLD image contrast at 7 Tesla. J Cereb Blood Flow Metab 20, 847-860.

Krieger, S.N., Ivanov, D., Huber, L., Roggenhofer, E., Sehm, B., Turner, R., Egan, G.F., Gauthier, C.J., 2014. Using carbogen for calibrated fMRI at 7Tesla: comparison of direct and modelled estimation of the M parameter. Neuroimage 84, 605-614.

Lauwers, F., Cassot, F., Lauwers-Cances, V., Puwanarajah, P., Duvernoy, H., 2008.

Morphometry of the human cerebral cortex microcirculation: general characteristics and spacerelated profiles. Neuroimage 39, 936-948.

Li, W., Wu, B., Batrachenko, A., Bancroft-Wu, V., Morey, R.A., Shashi, V., Langkammer, C., De Bellis, M.D., Ropele, S., Song, A.W., Liu, C., 2014. Differential developmental trajectories of magnetic susceptibility in human brain gray and white matter over the lifespan. Hum Brain Mapp 35, 2698-2713.

Liu, T., Wisnieff, C., Lou, M., Chen, W., Spincemaille, P., Wang, Y., 2013. Nonlinear formulation of the magnetic field to source relationship for robust quantitative susceptibility mapping. Magn Reson Med 69, 467-476.

Mark, C.I., Fisher, J.A., Pike, G.B., 2011. Improved fMRI calibration: precisely controlled hyperoxic versus hypercapnic stimuli. Neuroimage 54, 1102-1111.

Mazerolle, E.L., Ma, Y., Sinclair, D., Pike, G.B., 2016. Impact of abnormal cerebrovascular reactivity on BOLD fMRI: a preliminary investigation of moyamoya disease. Clin Physiol Funct Imaging.

Mazziotta, J., Toga, A., Evans, A., Fox, P., Lancaster, J., Zilles, K., Woods, R., Paus, T., Simpson, G., Pike, B., Holmes, C., Collins, L., Thompson, P., MacDonald, D., Iacoboni, M., Schormann, T., Amunts, K., Palomero-Gallagher, N., Geyer, S., Parsons, L., Narr, K., Kabani, N., Le Goualher, G., Boomsma, D., Cannon, T., Kawashima, R., Mazoyer, B., 2001. A 
probabilistic atlas and reference system for the human brain: International Consortium for Brain Mapping (ICBM). Philos Trans R Soc Lond B Biol Sci 356, 1293-1322.

Miller, K.L., Jezzard, P., 2008. Modeling SSFP functional MRI contrast in the brain. Magn Reson Med 60, 661-673.

Mugler, J.P., 3rd, Brookeman, J.R., 1990. Three-dimensional magnetization-prepared rapid gradient-echo imaging (3D MP RAGE). Magn Reson Med 15, 152-157.

Mulkern, R.V., Balasubramanian, M., Mitsouras, D., 2014. On the lorentzian versus Gaussian character of time-domain spin-echo signals from the brain as sampled by means of gradientechoes: Implications for quantitative transverse relaxation studies. Magn Reson Med.

Ni, W., Christen, T., Zun, Z., Zaharchuk, G., 2014. Comparison of R2' measurement methods in the normal brain at 3 tesla. Magn Reson Med.

Ogawa, S., Menon, R.S., Tank, D.W., Kim, S.G., Merkle, H., Ellermann, J.M., Ugurbil, K., 1993. Functional brain mapping by blood oxygenation level-dependent contrast magnetic resonance imaging. A comparison of signal characteristics with a biophysical model. Biophys $\mathrm{J}$ 64, 803-812.

Ordidge, R.J., Gorell, J.M., Deniau, J.C., Knight, R.A., Helpern, J.A., 1994. Assessment of relative brain iron concentrations using T2-weighted and T2*-weighted MRI at 3 Tesla. Magn Reson Med 32, 335-341.

Pannetier, N.A., Debacker, C.S., Mauconduit, F., Christen, T., Barbier, E.L., 2013. A simulation tool for dynamic contrast enhanced MRI. PLoS One 8, e57636.

Pannetier, N.A., Sohlin, M., Christen, T., Schad, L., Schuff, N., 2014. Numerical modeling of susceptibility-related MR signal dephasing with vessel size measurement: Phantom validation at 3T. Magn Reson Med 72, 646-658.

Pawlik, G., Rackl, A., Bing, R.J., 1981. Quantitative capillary topography and blood flow in the cerebral cortex of cats: an in vivo microscopic study. Brain Res 208, 35-58.

Sakadzic, S., Mandeville, E.T., Gagnon, L., Musacchia, J.J., Yaseen, M.A., Yucel, M.A., Lefebvre, J., Lesage, F., Dale, A.M., Eikermann-Haerter, K., Ayata, C., Srinivasan, V.J., Lo, E.H., Devor, A., Boas, D.A., 2014. Large arteriolar component of oxygen delivery implies a safe margin of oxygen supply to cerebral tissue. Nature Communications 5, 5734.

Sedlacik, J., Boelmans, K., Lobel, U., Holst, B., Siemonsen, S., Fiehler, J., 2014. Reversible, irreversible and effective transverse relaxation rates in normal aging brain at 3T. Neuroimage 84, 1032-1041.

Severinghaus, J.W., 1979. Simple, accurate equations for human blood O2 dissociation computations. J Appl Physiol 46, 599-602. 
Shu, C.Y., Herman, P., Coman, D., Sanganahalli, B.G., Wang, H., Juchem, C., Rothman, D.L., de Graaf, R.A., Hyder, F., 2015. Brain region and activity-dependent properties of $\mathrm{M}$ for calibrated fMRI. Neuroimage 125, 848-856.

Shu, C.Y., Sanganahalli, B.G., Coman, D., Herman, P., Rothman, D.L., Hyder, F., 2016. Quantitative beta mapping for calibrated fMRI. Neuroimage 126, 219-228.

Simon, A.B., Dubowitz, D.J., Blockley, N.P., Buxton, R.B., 2016. A novel Bayesian approach to accounting for uncertainty in fMRI-derived estimates of cerebral oxygen metabolism fluctuations. Neuroimage 129, 198-213.

Smith, S.M., 2002. Fast robust automated brain extraction. Hum Brain Mapp 17, 143-155.

Spees, W.M., Yablonskiy, D.A., Oswood, M.C., Ackerman, J.J., 2001. Water proton MR properties of human blood at 1.5 Tesla: magnetic susceptibility, T(1), T(2), T*(2), and nonLorentzian signal behavior. Magn Reson Med 45, 533-542.

Stefanovic, B., Hutchinson, E., Yakovleva, V., Schram, V., Russell, J.T., Belluscio, L., Koretsky, A.P., Silva, A.C., 2008. Functional reactivity of cerebral capillaries. J Cereb Blood Flow Metab 28, 961-972.

Stone, A.J., Blockley, N.P., 2016. A streamlined acquisition for mapping baseline brain oxygenation using quantitative BOLD. Neuroimage 147, 79-88.

Stuber, C., Morawski, M., Schafer, A., Labadie, C., Wahnert, M., Leuze, C., Streicher, M., Barapatre, N., Reimann, K., Geyer, S., Spemann, D., Turner, R., 2014. Myelin and iron concentration in the human brain: a quantitative study of MRI contrast. Neuroimage 93 Pt 1, 95106.

Sukstanskii, A.L., Yablonskiy, D.A., 2003. Gaussian approximation in the theory of MR signal formation in the presence of structure-specific magnetic field inhomogeneities. J Magn Reson $163,236-247$.

Tancredi, F.B., Lajoie, I., Hoge, R.D., 2014. A simple breathing circuit allowing precise control of inspiratory gases for experimental respiratory manipulations. BMC Res Notes 7, 235.

Turner, R., 2002. How much cortex can a vein drain? Downstream dilution of activation-related cerebral blood oxygenation changes. Neuroimage 16, 1062-1067.

Uludag, K., Dubowitz, D.J., Yoder, E.J., Restom, K., Liu, T.T., Buxton, R.B., 2004. Coupling of cerebral blood flow and oxygen consumption during physiological activation and deactivation measured with fMRI. Neuroimage 23, 148-155.

Wang, Z., 2012. Improving cerebral blood flow quantification for arterial spin labeled perfusion MRI by removing residual motion artifacts and global signal fluctuations. Magn Reson Imaging $30,1409-1415$. 
Wansapura, J.P., Holland, S.K., Dunn, R.S., Ball, W.S., 1999. NMR relaxation times in the human brain at 3.0 tesla. J Magn Reson Imaging 9, 531-538.

Weiskopf, N., Hutton, C., Josephs, O., Turner, R., Deichmann, R., 2007. Optimized EPI for fMRI studies of the orbitofrontal cortex: compensation of susceptibility-induced gradients in the readout direction. MAGMA 20, 39-49.

Xu, F., Uh, J., Brier, M.R., Hart, J., Jr., Yezhuvath, U.S., Gu, H., Yang, Y., Lu, H., 2011. The influence of carbon dioxide on brain activity and metabolism in conscious humans. J Cereb Blood Flow Metab 31, 58-67.

Yablonskiy, D.A., 1998. Quantitation of intrinsic magnetic susceptibility-related effects in a tissue matrix. Phantom study. Magn Reson Med 39, 417-428.

Yablonskiy, D.A., Haacke, E.M., 1994. Theory of NMR signal behavior in magnetically inhomogeneous tissues: the static dephasing regime. Magn Reson Med 32, 749-763.

Zhao, F., Wang, P., Kim, S.G., 2004. Cortical depth-dependent gradient-echo and spin-echo BOLD fMRI at 9.4T. Magn Reson Med 51, 518-524. 


\section{Supplementary Material}

\section{Gas-free Calibrated fMRI with a Correction for Vessel-Size Sensitivity}

Avery J.L. Berman, Erin L. Mazerolle, M. Ethan MacDonald, Nicholas P. Blockley, Wen-Ming

Luh, G. Bruce Pike

\section{S1. q-ASE Model Assumptions}

\section{S1.1 Quadratic-to-Linear Transition}

To determine whether the spin echo attenuation displayed in Fig. 3 was best described by linearexponential or quadratic-exponential decay, $\log \left(S_{\mathrm{ASE}}\right)$ was fit vs. TE to a linear polynomial (fitting for an intercept and slope) and to the q-ASE model (fitting for $R_{2}{ }^{\prime}$ and $\left.\left(R_{2, \text { diff }}\right)^{2}\right)$. The two models were fit using a sliding window of width $10 \mathrm{~ms}$, starting at TE $=\tau$, and shifting in 2-ms increments. The quality of fit at each window position was assessed using the coefficient of determination $\left(\mathrm{R}^{2}\right)$. The ratio of the q-ASE to linear $\mathrm{R}^{2}$ values was calculated such that the $\mathrm{q}$ ASE model outperformed the linear model if the ratio was greater than 1.

The $\mathrm{R}^{2}$ ratios are shown in Fig. S1 as a function of TE for four $\tau$ values. The decrease of this ratio over time shows the transition from quadratic to linear decay. All the $\mathrm{R}^{2}$ ratios were greater than or approximately equal to 1 . To show how the time to transition from quadratic to linear decay depends on vessel size, the time where the $\mathrm{R}^{2}$ ratio drops below a threshold of 1.01 is plotted in Fig. S2. This figure indicates that the time to transition increases with vessel radius up to approximately $20 \mu \mathrm{m}$. Beyond $20 \mu \mathrm{m}$, the transition time slowly decreases (bearing in mind the semi-logarithmic scale). Excluding the $\tau=0$ transition time curve, the curves are approximately shifted versions of each (vertically shifted by $\tau$ ), further supporting the observation in Fig. 3 that the ASE decays for a given radius are shifted versions of each other from $\tau$-to- $\tau$. 

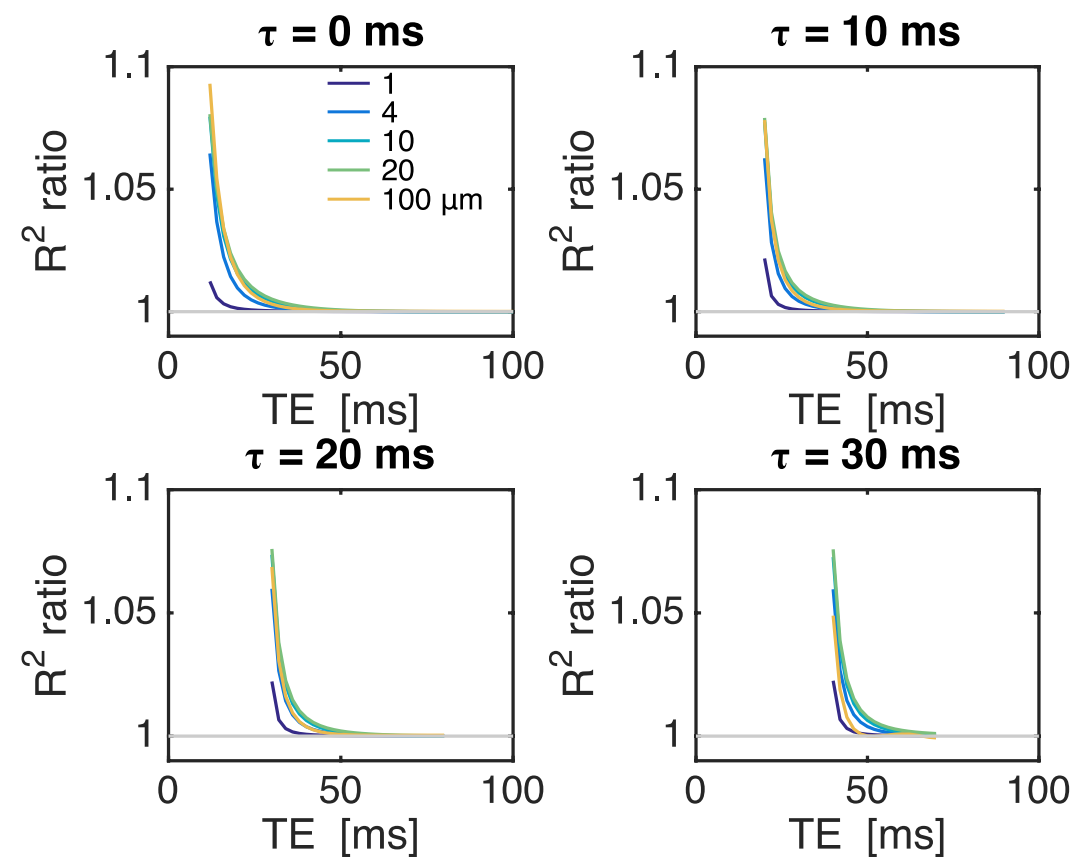

Fig. S1: The ratio of the coefficients of determination $\left(\mathrm{R}^{2}\right)$ of the q-ASE fit vs. the linear fit to $\log \left(S_{\mathrm{ASE}}\right)$. The grey line shows where the ratio equals 1 . For each ASE offset, curves begin at TE $=\tau+10 \mathrm{~ms}$ (the sliding window width).

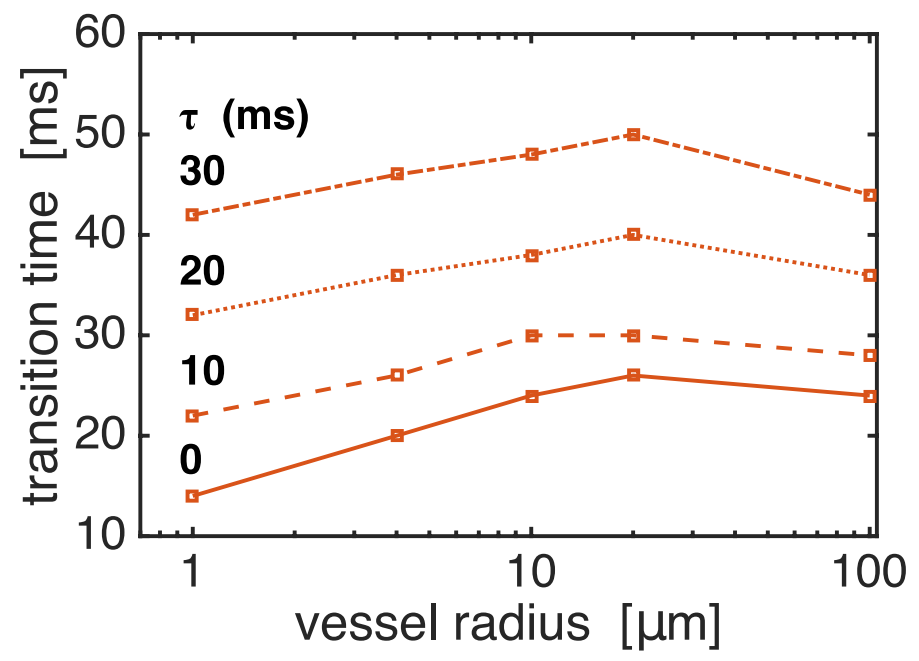

Fig. S2: The quadratic to linear decay transition times for each ASE offset. Transition times are defined as the time where the $\mathrm{R}^{2}$ ratios from Fig. S1 are less than 1.01. Each ASE offset is labeled on the left and plotted with a different line style.

\section{S1.2 q-ASE Fit Parameters}


To further verify the validity of the q-ASE model, it was fit to $\log \left(S_{\mathrm{ASE}}\right)$ vs. TE from TE $=\tau$ to $\mathrm{TE}=\tau+30 \mathrm{~ms}$. This was performed at every possible $\tau$ value, giving the dependence of $R_{2}{ }^{\prime}$ and $\left(R_{2, \text { diff }}\right)^{2}$ on $\tau$. If the $\log \left(S_{\mathrm{ASE}}\right)$ curves are shifted versions of each other, $\left(R_{2, \text { diff }}\right)^{2}$ should be constant, as it dictates the curvature of the quadratic. $R_{2}{ }^{\prime}$ is expected to vary with $\tau$ until it plateaus at some value since it is known that $T_{2}{ }^{*}$ decay asymptotically approaches monoexponential decay (Yablonskiy and Haacke, 1994).

Fig. S3 shows the results of these fits. $\left(R_{2, \text { diff }}\right)^{2}$ is not constant across $\tau$, although it does change slowly. This variation consists of an increase to some peak value followed by a decrease. Excluding the $1-\mu \mathrm{m}$ simulations, the magnitude of $\left(R_{2, \text { diff }}\right)^{2}$ monotonically decreases with radius, consistent with Fig. 7 , for all $\tau$ values. The rise and fall of $\left(R_{2, \text { diff }}\right)^{2}$ also appears to occur more rapidly as the vessel size increases.
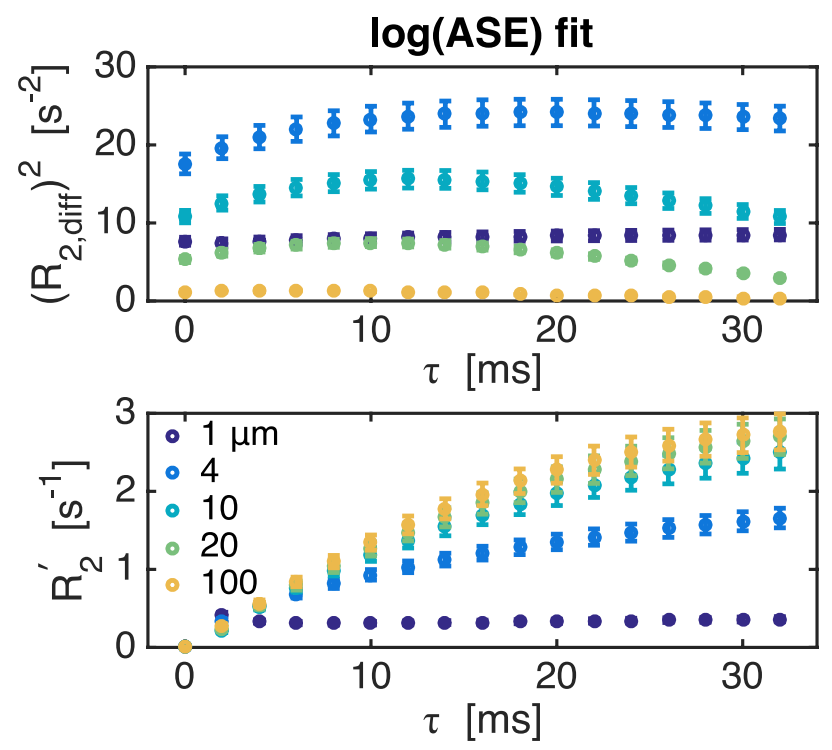

Fig. S3: The $\left(R_{2, d i f f}\right)^{2}$ (top) and $R_{2}{ }^{\prime}$ (bottom) values estimated from the simulations when $\log \left(S_{\mathrm{ASE}}\right)$ vs. TE was fit to the q-ASE model at each ASE offset $(\tau)$. Markers represent the mean across all networks \pm the standard deviation. Each colour represents a different vessel radius, specified in the bottom figure's legend.

\section{S1.3 q-ASE Log-Ratio Fit Parameters}


In practice, the ratios of the SE and ASE signals are used when estimating $R_{2}{ }^{\prime}$ since this removes the $T_{2}$-dependence of the individual signals. From Eq. (7), the q-ASE model predicts that the logarithm of that ratio has a linear dependence on TE when $\tau$ is held fixed. This ratio was therefore fit from TE $=\tau$ to $\mathrm{TE}=\tau+30 \mathrm{~ms}$ at each $\tau$ value to see how the estimates of $R_{2}{ }^{\prime}$ and $\left(R_{2, \text { diff }}\right)^{2}$ depend on $\tau$. The degree to which the log-ratio showed the linear decay was quantified using the coefficient of determination.

The fit results are shown in Fig. S4 along with the percent difference between the $\left(R_{2, \text { diff }}\right)^{2}$ and $R_{2}{ }^{\prime}$ estimates obtained from the log-ratio and from the ASE signals, alone, in Fig. S3. In Fig. $\mathrm{S} 4(\mathrm{~b})$, the $\left(R_{2, \text { diff }}\right)^{2}$ estimates vary massively between the two different fittings. Despite this, excluding the two smallest vessel sizes, $R_{2}{ }^{\prime}$ agreed within $\pm 5 \%$ between the two fittings. In the scope of calibrated fMRI, this latter result is of prime importance since $R_{2}{ }^{\prime}$ alone dictates the $M$ value when calculated using Eq. (8). Finally, it can be seen how the $\mathrm{R}^{2}$ values of the smaller vessels are lowest and gradually increase. The increase of the $\mathrm{R}^{2}$ values reflects the transition from the quadratic to linear decay regimes, where the $\log \left(S_{\mathrm{SE}} / S_{\mathrm{ASE}}\right)$ points do not lie along a straight line while transitioning (see Fig. 8).

The discrepancy between the $\left(R_{2, d i f f}\right)^{2}$ estimates is surprising, however, given that the $R_{2}{ }^{\prime}$ estimates are sufficiently accurate, this discrepancy will be considered in future studies. Given that the log-ratio fitting is what would typically be performed experimentally, we refer to its estimates of $\left(R_{2, \text { diff }}\right)^{2}$ and $R_{2}^{\prime}$ as their apparent values. 
(a)

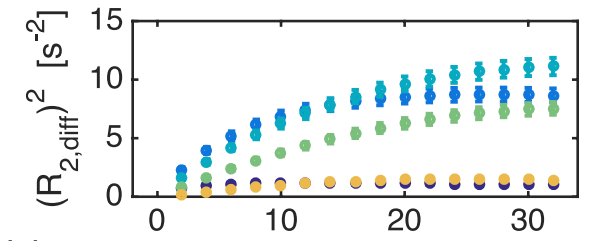

(c)

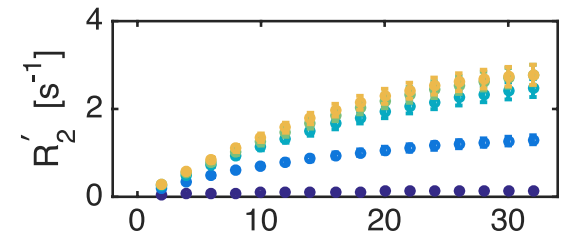

(e)

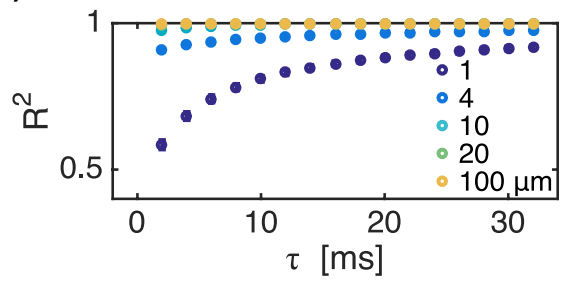

(b) $\quad \log (\mathrm{SE} / \mathrm{ASE})-\log (\mathrm{ASE}) \%$ Diff.

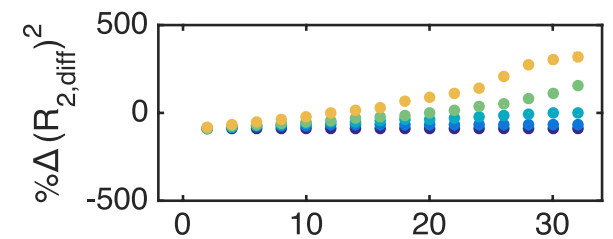

(d)

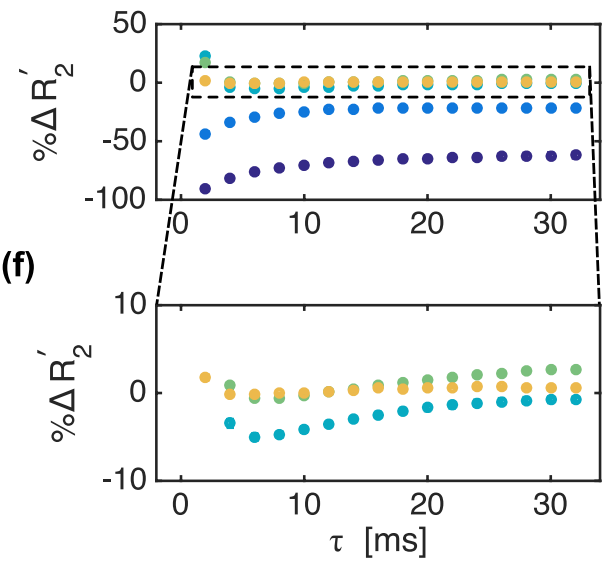

Fig. S4: The $\left(R_{2, d i f f}\right)^{2}$ (a) and $R_{2}{ }^{\prime}(\mathrm{c})$ values estimated from the simulations when the log-ratio, $\log \left(S_{\mathrm{SE}} / S_{\mathrm{ASE}}\right)$, vs. TE was fit linearly to the q-ASE model at each ASE offset $(\tau)$. The coefficients of determination $\left(\mathrm{R}^{2}\right)$ at each $\tau$ for all vessel sizes are plotted in (e). The percent difference between the $\left(R_{2, d i f f}\right)^{2}$ and $R_{2}{ }^{\prime}$ values estimated from the log-ratio vs. estimated from the raw signals themselves $\left(\log \left(S_{\mathrm{ASE}}\right)\right)$ are plotted in (b) and (d). (f) shows a zoom-in of the $R_{2}{ }^{\prime}$ differences within the range enclosed in the dashed black box in (d). Each colour represents a different vessel radius, specified in the legend in (e).

\section{S2. Physiological Variability}

The applicability of the q-ASE model to a range of physiological parameters was assessed by simulating the ASE signals with multiple venous cerebral blood volumes (CBV) and blood-tissue susceptibility offsets $(\Delta \chi)$. The preliminary simulations used $\mathrm{CBV}=2 \%$ and $\Delta \chi=4 \times 10^{-8}$, where this $\Delta \chi$ approximately corresponds to the susceptibility offset when blood oxygen saturation $\left(\mathrm{SO}_{2}\right)=60 \%$ and hematocrit $(\mathrm{Hct})=40 \%$. The simulations were repeated with $\mathrm{CBV}=4$ and $6 \%$, while $\Delta \chi$ was held constant at $4 \times 10^{-8}$. The effect of $\Delta \chi$ was probed with two additional values by considering the range of plausible venous $\mathrm{SO}_{2}$ to be $50-70 \%$ and Hct to be $35-50 \%$. The simulations were then run with the combination of $\mathrm{SO}_{2}$ and Hct that produced the extreme largest and smallest susceptibility offsets while CBV was held constant at $2 \%$. Those 
combinations were $\mathrm{SO}_{2}=50 \%$ and $\mathrm{Hct}=50 \%\left(\Delta \chi=6.6 \times 10^{-8}\right)$ and $\mathrm{SO}_{2}=70 \%$ and $\mathrm{Hct}=35 \%$ $\left(\Delta \chi=2.8 \times 10^{-8}\right)$.

$\left(R_{2, \text { diff }}\right)^{2}$ and $R_{2}{ }^{\prime}$ were found to have linear dependences on CBV in all the comparisons examined and these dependences were the same for all vessel radii. As a result, the quadratic to linear decay transition times were virtually unchanged between all CBVs. Fig. S5(a) and (c) show the linear CBV dependence of the apparent $\left(R_{2, \text { diff }}\right)^{2}$ and $R_{2}{ }^{\prime}$ values when obtained from the $\log$-ratio fits at $\tau=30 \mathrm{~ms}$.

(a)

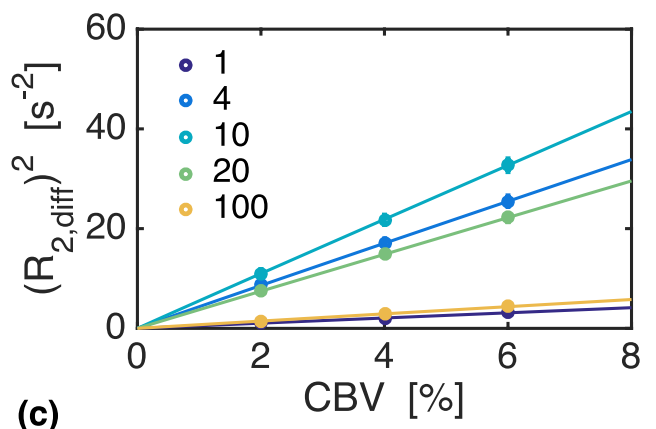

(c)

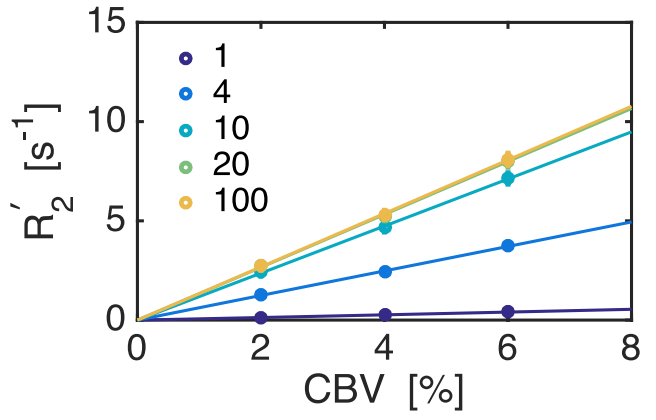

(b)

$\Delta \chi$ Dependence
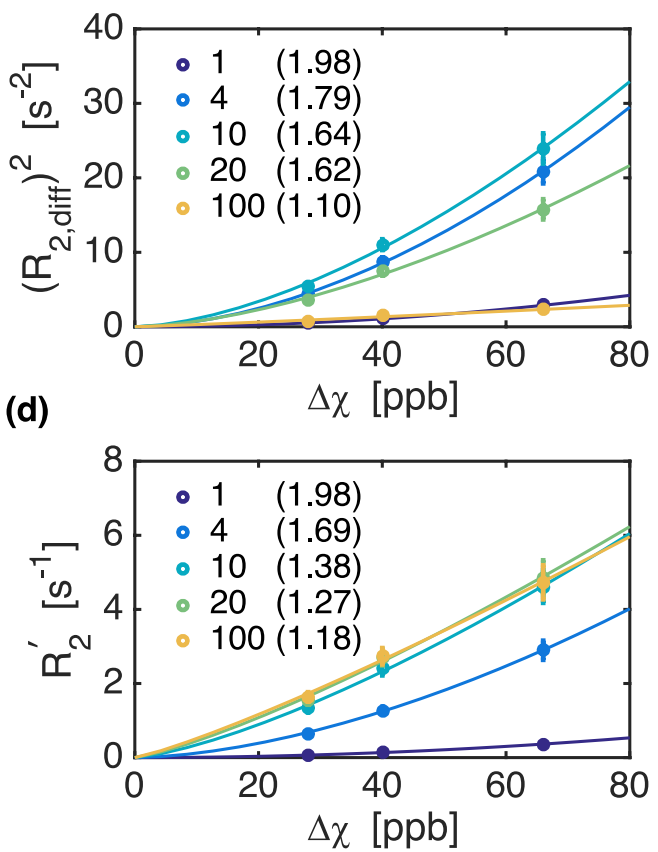

Fig. S5: The CBV and $\Delta \chi$ dependences of the apparent $\left(R_{2, \text { diff }}\right)^{2}$ and $R_{2}{ }^{\prime}$ values at $\tau=30 \mathrm{~ms}$. The CBV dependences of both $\left(R_{2, \text { diff }}\right)^{2}(\mathrm{a})$ and $R_{2}{ }^{\prime}(\mathrm{c})$ are linear. The $\Delta \chi$ dependences of $\left(R_{2, \text { diff }}\right)^{2}(\mathrm{~b})$ and $R_{2}^{\prime}(\mathrm{d})$ are both non-linear and depend on vessel size. Legends in each figure show the radii, and the values in parentheses are the exponents relating $\left(R_{2, \text { diff } f}\right)^{2}$ and $R_{2}{ }^{\prime}$ to $\Delta \chi$ for each radius.

The $\Delta \chi$ dependence of $\left(R_{2, \text { diff }}\right)^{2}$ and $R_{2}^{\prime}$ was non-linear and varied between vessel radii, as shown in Fig. S5(b) and (c). These dependences were fit to functions of the form $y=a(\Delta \chi)^{b}$, where, in the case of $R_{2}{ }^{\prime}, b$ is the same as $\beta$ used in the calibrated fMRI model. The fitted $b$ values are listed in the figure legends. 
The times to transition from quadratic to linear decay also changed with $\Delta \chi$, as shown in Fig. S6. For the earlier $\tau$ 's, the transition times generally decreased slightly with $\Delta \chi$, whereas, at the later $\tau$ 's, the times were a bit more variable and increased slightly with $\Delta \chi$. The overall trend being that the transition time increases with vessel radius and either plateaus or decreases beyond a given radius. With increasing vessel radius, the simulations approach that of no diffusion, where neither quadratic nor linear decay is exhibited. In this case, it is unclear whether the transition time should be asymptotically approaching an infinite value or zero. Intuitively, we believe it is the former since it will take longer to accrue irreversible phase through diffusion as the vessel size increases, therefore, extending the transition time. Practically, however, the transition time of the largest vessels is unimportant as refocusing is sufficient to accurately estimate $R_{2}^{\prime}$.

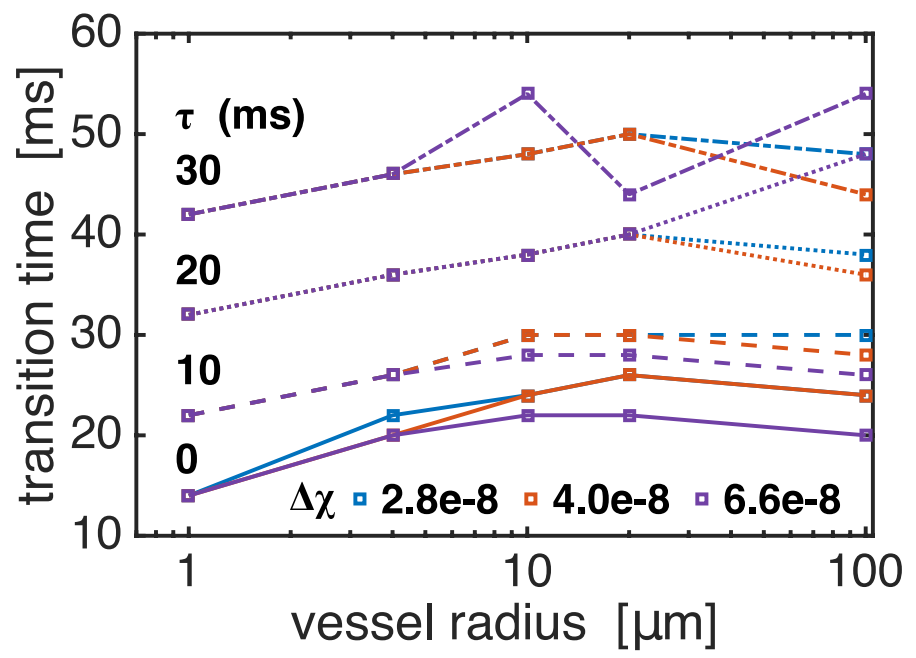

Fig. S6: The quadratic to linear decay transition times for each ASE offset and each susceptibility offset $(\Delta \chi)$. Transition times are defined as the time where the $\mathrm{R}^{2}$ ratios of the qASE to linear fits are less than 1.01. Each ASE offset is labeled on the left and plotted with a different line type. Each $\Delta \chi$ is plotted with a different colour, following the legend along the bottom.

\section{S3. Impact of Signal-to-Noise Ratio}


The impact of signal-to-noise ratio (SNR) on the estimates of $\left(R_{2, \text { diff }}\right)^{2}$ and $M$ was examined using the signal model with intra- and extra-vascular $T_{2}$ decay described in section 3.2.1. Gaussian white noise was added to the simulated signals from the Lauwers and Frechet networks, such that the signal at $\mathrm{TE} / \tau=40 / 30 \mathrm{~ms}$ would have the desired SNR. SNR values ranged from 100 to 400 with 1000 randomizations of each noise level.

These results are summarized in Fig. S7 and Fig. S8 for the Lauwers and Frechet distributions, respectively. For both distributions, figures (a)-(d) show that a very high SNR is required to preserve the true individual networks' log-ratios and the slopes (proportional to $\left.\left(R_{2, \text { diff }}\right)^{2}\right)$ vary considerably. The net result is that the error bars on the fitted $\left(R_{2, \text { diff }}\right)^{2}$ and $M$ values rapidly grow as SNR decreases. Despite this increase in variability, the mean fitted values were relatively unchanged.

For comparison to the in vivo results, after motion correction of the in vivo acquisitions, the mean over the standard deviation across time (i.e., time-SNR) of the $\mathrm{TE} / \tau=42 / 30 \mathrm{~ms}$ images was approximately 40 to 50 in grey matter. Since the 15 repetitions were averaged across time, the resulting SNR was $\sim 150-200(=40-50 \times \sqrt{ } 15)$. The SNR of the in vivo acquisitions was further increased since the signals were averaged across regions of interest. Therefore, the in vivo SNR was likely a minimum of 150 . 

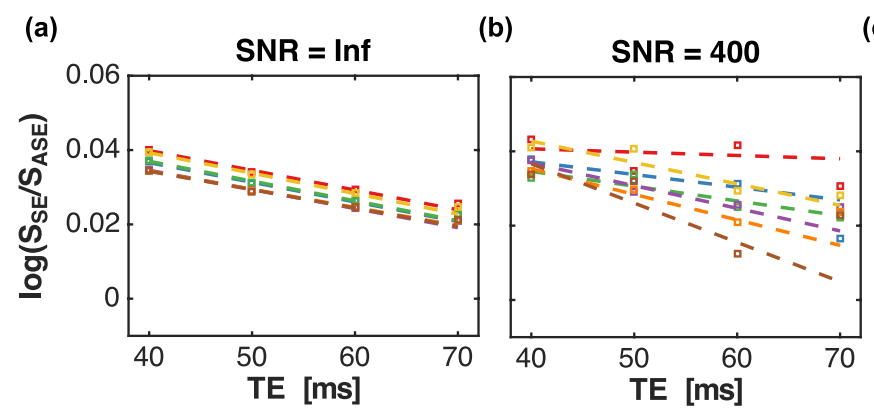

(c)

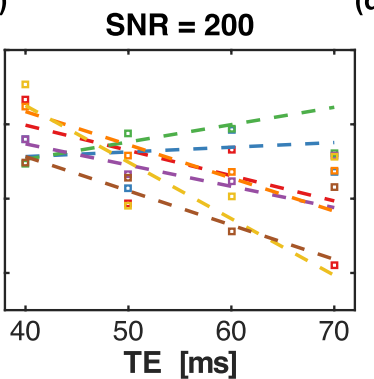

(d)

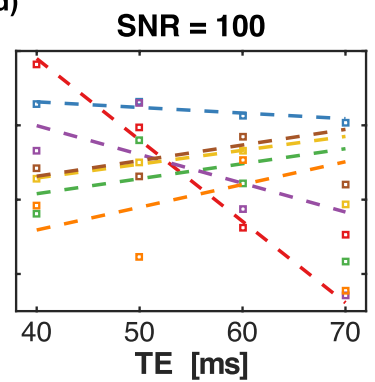

(e)

M vs. SNR

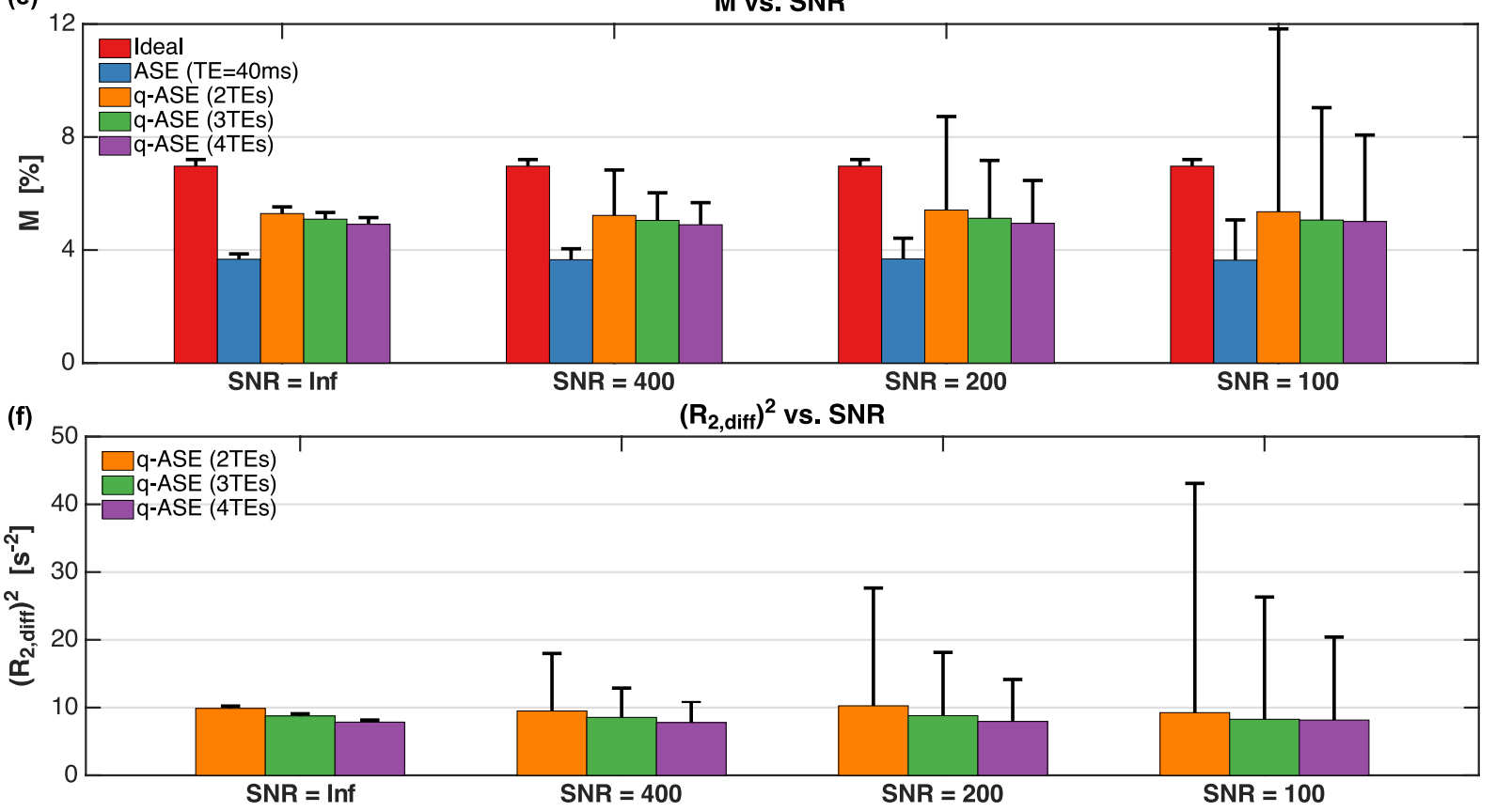

Fig. S7: Impact of signal-to-noise ratio (SNR) on $\log \left(S_{\mathrm{SE}} / S_{\mathrm{ASE}}\right)(\mathrm{a})-(\mathrm{d})$ and the resulting estimates of $M(\mathrm{e})$ and $\left(R_{2, \text { diff }}\right)^{2}$ (f) for the Lauwers vessel size distribution. (a)-(d) show examples of the ASE log-ratio, with each coloured symbol corresponding to simulations from a different vessel network and different instantiation of noise. Dashed lines correspond to the predicted ratios when $\left(R_{2, \text { diff }}\right)^{2}$ and $R_{2}{ }^{\prime}$ were fit using the first three echo times (TE $=40$ to 60 $\mathrm{ms}$ ). The bar plots in (e) and (f) show the mean fitted values and the error bars correspond to the standard deviation. SNR = Inf corresponds to the case where no noise was added to the simulations and the variability arises from the inherent variation from simulation network to network. In all cases, no noise was added to the reference simulations ( $M_{\text {ideal, }}$, red bars). 

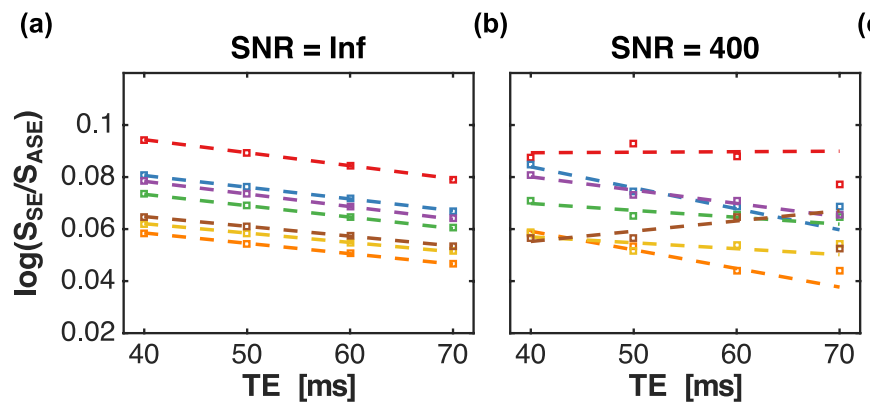

(c)

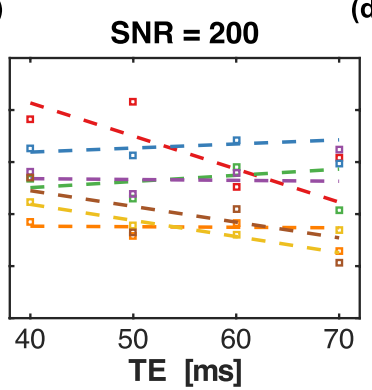

(d)

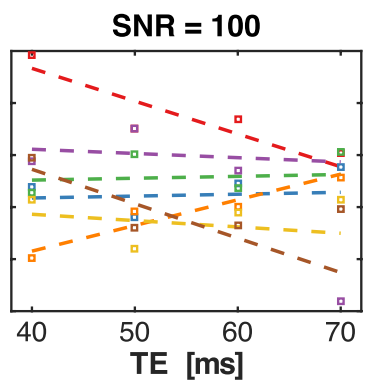

(e)

M vs. SNR

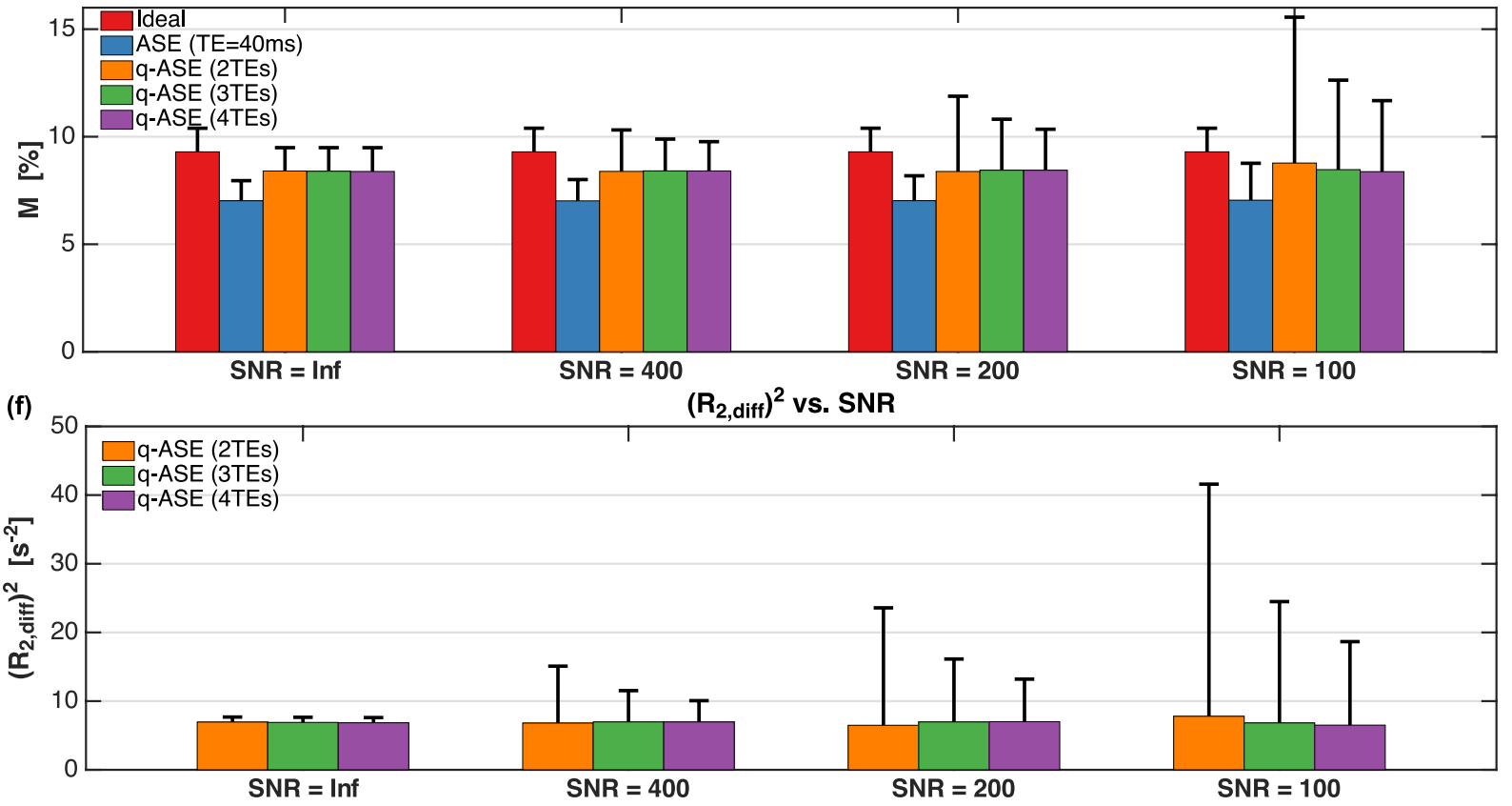

Fig. S8: Impact of signal-to-noise ratio (SNR) on $\log \left(S_{\mathrm{SE}} / S_{\mathrm{ASE}}\right)($ a) $)(\mathrm{d})$ and the resulting estimates of $M(\mathrm{e})$ and $\left(R_{2, \text { diff }}\right)^{2}$ (f) for the Frechet vessel size distribution. (a)-(d) show examples of the ASE log-ratio, with each coloured symbol corresponding to simulations from a different vessel network and different instantiation of noise. Dashed lines correspond to the predicted ratios when $\left(R_{2, \text { diff }}\right)^{2}$ and $R_{2}{ }^{\prime}$ were fit using the first three echo times (TE $=40$ to $60 \mathrm{~ms}$ ). The bar plots in (e) and (f) show the mean fitted values and the error bars correspond to the standard deviation. SNR = Inf corresponds to the case where no noise was added to the simulations and the variability arises from the inherent variation from simulation network to network. In all cases, no noise was added to the reference simulations ( $M_{\text {ideal, }}$, red bars). 


\section{References}

Yablonskiy, D.A., Haacke, E.M., 1994. Theory of NMR signal behavior in magnetically inhomogeneous tissues: the static dephasing regime. Magn Reson Med 32, 749-763. 